
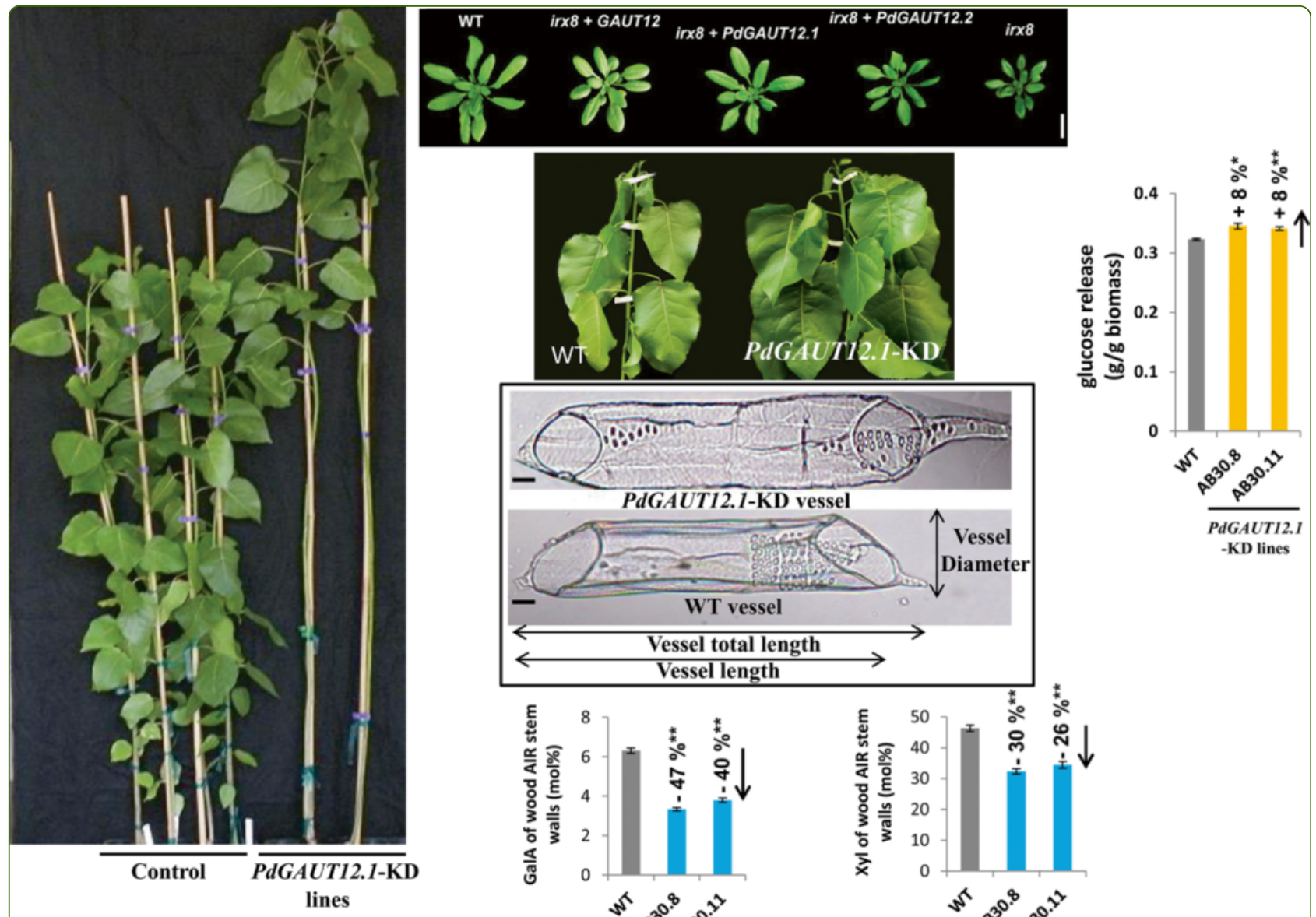

Downregulation of GAUT12 in Populus deltoides by RNA silencing results in reduced recalcitrance, increased growth and reduced xylan and pectin in a woody biofuel feedstock

Biswal et al. 


\title{
Downregulation of GAUT12 in Populus deltoides by RNA silencing results in reduced recalcitrance, increased growth and reduced xylan and pectin in a woody biofuel feedstock
}

Ajaya K Biswal 1,3,4, Zhangying Hao ${ }^{2,3,4}$, Sivakumar Pattathil ${ }^{3,4}$, Xiaohan Yang ${ }^{4,5}$, Kim Winkeler ${ }^{4,6}$, Cassandra Collins ${ }^{4,6}$, Sushree S Mohanty 3,4, Elizabeth A Richardson², Ivana Gelineo-Albersheim, ${ }^{3,4}$ Kimberly Hunt ${ }^{3,4}$, David Ryno 3,4, Robert W Sykes ${ }^{4,7}$, Geoffrey B Turner ${ }^{4,7}$, Angela Ziebell ${ }^{4,7}$, Erica Gjersing ${ }^{4,7}$, Wolfgang Lukowitz ${ }^{2}$, Mark F Davis ${ }^{4,7}$, Stephen R Decker ${ }^{4,7}$, Michael G Hahn $n^{2,3,4}$ and Debra Mohnen ${ }^{1,3,4^{*}}$

\begin{abstract}
Background: The inherent recalcitrance of woody bioenergy feedstocks is a major challenge for their use as a source of second-generation biofuel. Secondary cell walls that constitute the majority of hardwood biomass are rich in cellulose, xylan, and lignin. The interactions among these polymers prevent facile accessibility and deconstruction by enzymes and chemicals. Plant biomass that can with minimal pretreatment be degraded into sugars is required to produce renewable biofuels in a cost-effective manner.

Results: GAUT12/IRX8 is a putative glycosyltransferase proposed to be involved in secondary cell wall glucuronoxylan and/or pectin biosynthesis based on concomitant reductions of both xylan and the pectin homogalacturonan (HG) in Arabidopsis irx8 mutants. Two GAUT12 homologs exist in Populus trichocarpa, PtGAUT12.1 and PtGAUT12.2. Knockdown expression of both genes simultaneously has been shown to reduce xylan content in Populus wood. We tested the proposition that RNA interference (RNAi) downregulation of GAUT12.1 alone would lead to increased sugar release in Populus wood, that is, reduced recalcitrance, based on the hypothesis that GAUT12 synthesizes a wall structure required for deposition of xylan and that cell walls with less xylan and/or modified cell wall architecture would have reduced recalcitrance. Using an RNAi approach, we generated 11 Populus deltoides transgenic lines with 50 to 67\% reduced PdGAUT12.1 transcript expression compared to wild type (WT) and vector controls. Ten of the eleven RNAi lines yielded 4 to $8 \%$ greater glucose release upon enzymatic saccharification than the controls. The PdGAUT12.1 knockdown (PdGAUT12.1-KD) lines also displayed 12 to 52\% and 12 to $44 \%$ increased plant height and radial stem diameter, respectively, compared to the controls. Knockdown of PdGAUT12.1 resulted in a 25 to $47 \%$ reduction in galacturonic acid and 17 to $30 \%$ reduction in xylose without affecting total lignin content, revealing that in Populus wood as in Arabidopsis, GAUT12 affects both pectin and xylan formation. Analyses of the sugars present in sequential cell wall extracts revealed a reduction of glucuronoxylan and pectic HG and rhamnogalacturonan in extracts from PdGAUT12.1-KD lines.

(Continued on next page)
\end{abstract}

\footnotetext{
* Correspondence: dmohnen@ccrc.uga.edu

'Department of Biochemistry and Molecular Biology, University of Georgia,

B122 Life Sciences Bldg., Athens, GA 30602, USA

${ }^{3}$ Complex Carbohydrate Research Center, University of Georgia, 315

Riverbend Road, Athens, GA 30602, USA

Full list of author information is available at the end of the article
} 
(Continued from previous page)

Conclusions: The results show that downregulation of GAUT12.1 leads to a reduction in a population of xylan and pectin during wood formation and to reduced recalcitrance, more easily extractable cell walls, and increased growth in Populus.

Keywords: Biofuel, Growth, Pectin, Populus, Saccharification, Secondary cell wall, Xylan, Wood development

\section{Background}

Populus is a woody feedstock for biofuel and bioproduct formation. The major challenge of using woody feedstock as a source for biofuels is the rigid cell wall, which is recalcitrant to degradation by bacterial and fungal enzymes [1-3]. The identification of genes and proteins involved in the formation of secondary cells wall is necessary to understand and overcome the recalcitrance of woody feedstocks. Towards this aim, we have manipulated the expression of putative 'recalcitrance' genes in Populus for use in studying the genetic basis of recalcitrance in this biomass feedstock.

Wood formation in Populus starts with the differentiation of secondary cell walls. Cellulose, hemicellulose, and lignin are the three major components of Populus secondary walls, with pectin being a minor component. In Populus wood, the hemicelluloses are largely xylans which provide 18 to $28 \%$ of the total dry weight [4]. Xylans are polysaccharides with linear backbones of $\beta$ $(1 \rightarrow 4)$-linked D-xylosyl residues. The major xylan in dicot wood, glucuronoxylan (GX), is decorated with side chains of $O$-2-linked $\alpha$-D-glucuronic acid (GlcA) and/or 4-O-methyl- $\alpha$-D-glucuronic acid (MeGlcA). The xylosyl backbone in secondary wall xylan is also highly acetylated at $\mathrm{C}-2$ and $\mathrm{C}-3$.

Multiple types of Arabidopsis xylan synthesis mutants [5], including xylan backbone mutants irxy irxy-L [6-9], irx14 irx14-L [9-11], irxx10 irx10-L [12,13], and irx15 $\operatorname{irx} 15-L[14,15]$, have been extensively studied in an effort to understand xylan biosynthesis. The recovery of xylan xylosyltransferase activity from heterologously expressed Arabidopsis IRX10-L [16] and Plantago ovate IRX10 [17], and the demonstration of xylan acetyltransferase activity from heterologously expressed Arabidopsis ESK1/TBL29 [16], confirmed a role for these enzymes in xylan backbone synthesis and acetylation, respectively. Arabidopsis xylan substitution mutants gux 1 and gux2 have reduced $\alpha$-glucuronidation of the xylan backbone $[18,19]$ while the level of methylation of the GlcA residues is reduced in gxmt1 mutants [20]. The respective genes have been shown to encode functional xylan glucuronosyltransferases [18] and xylan 4$O$-methyltransferases [20]. Additional Arabidopsis mutants have also been identified that have defects in both xylan and other cell wall polymers. For example, xylan and cellulose deposition are affected in $i r x 7$ (fra8) and irx7-L (F8H) [10,21,22] while qua1 [23-25], parvus-3 [10,26-28], and irx8/GAUT12 [8,29,30] have defects in both pectin and xylan.

The Arabidopsis irx 8 mutant has been extensively characterized in Arabidopsis [5]. The IRX8/GAUT12 gene belongs to the GAUT1 (GAlactUronosylTransferase1)related gene family. The GAUTs constitute one clade of the glycosyltransferase 8 (GT8) family [30-33]. The family name, GAUT, originated with the identification of Arabidopsis galacturonosyltransferase 1 (GAUT1). GAUT1 is a pectin biosynthetic homogalacturonan (HG): $\alpha$-1,4-galacturonosyltransferase (GalAT) that functions in an HG:GalAT protein complex with GAUT7 [34-36]. Arabidopsis GAUT12/irx8 has highest expression in cells with secondary walls, and the encoded protein has $61 \%$ amino acid sequence similarity with GAUT1. GAUT12 is predicted to be a type II membrane protein targeted to the Golgi. The irx8/gaut12 mutation leads to a reduction in GX; however, microsomes from irx 8 mutant stems did not show any reduction in xylan XylT activity $[7,10]$ or xylan GlcAT (glucuronosyltransferase) activity [7] compared to microsomes from wild type (WT). Structural analysis of cell walls from irx8 Arabidopsis mutant plants identified a dramatic reduction in GX and in a tetrasaccharide sequence $\beta$-D-Xyl $p$-(1-3)- $\alpha$-L-Rhap-(1-2)$\alpha-\mathrm{D}-\mathrm{Gal} p \mathrm{~A}-(1-4)-\mathrm{D}-\mathrm{X} y \mathrm{p}$ located at the reducing end of xylan $[8,29]$. A concomitant reduction in a subfraction of the pectin HG was also identified in walls of Arabidopsis irx8 mutants compared to WT leading to the hypotheses that GAUT12 is involved in either xylan or HG synthesis [29].

Populus has two orthologs of the Arabidopsis GAUT12 gene: PtGAUT12.1 (POPTR_0001s44250, Genemodel V2.0, Phytozome 8.0, http://www.phytozome.net; Potri. 001G416800, Genemodel V3.0, Phytozome 10.0, http:// phytozome.jgi.doe.gov) and PtGAUT12.2 (POPTR 0011s13600, Genemodel V2.0, Phytozome 8.0; Potri.011G132600, Genemodel V3.0, Phytozome 10.0). Downregulation of both genes simultaneously in transgenic $P$. trichocarpa RNA interference (RNAi) lines (PtrGT8D1/POPTR_0001s44250/Potri.001G416800 and PtrGT8D2/POPTR_0011s13600/Potri.011G132600) led to a major reduction in xylan and an increase in lignin in the stem, along with reduced wall thickness in fiber cells [37]. Based on these results, a role for PtrGT8D in GX biosynthesis in Populus wood has been 
suggested [37]. The irregular xylem and dwarf phenotype observed in the Arabidopsis irx 8 mutant, however, was not observed in these Populus transgenic RNAi lines. In another study, it was reported that overexpression of full-length PoGT8D, the Populus alba $x$ tremula homolog of POPTR_0001s44250/Potri.001G416800 (Phytozome 8.0/Phytozome 10.0), did not complement the Arabidopsis irx8 mutant [38], although RNAi downregulation of PoGT8D suggested a slight reduction in the amount of xylan reducing end sequence [39]. The PoGT8D protein was shown to be targeted to the Golgi, matching its predicted type II membrane topology and in agreement with its involvement in the biosynthesis of non-cellulosic polysaccharides in Populus wood. Since the biochemical activity of the GAUT12 protein remains to be determined, it is not clear how this protein is involved in GX biosynthesis in Populus. Both of the GAUT12 Populus trichocarpa genes are expressed in primary xylem, differentiating xylem, secondary xylem, and phloem fibers in the woody stem. The expression of PtrGT8D1 has been reported to be seven times greater than PtrGT8D2 [37,38].

The recalcitrance of Populus biomass to deconstruction is the major obstacle to the bioconversion of this lignocellulosic feedstock into biofuels [40]. Heavily acetylated xylan cannot be efficiently hydrolyzed [41] and xylose, xylan, and xylooligomers, when produced during saccharification, inhibit hydrolysis of cellulose by cellulase as well as conversion rates and yields [42]. Reducing xylan levels in Populus is, therefore, one strategy to reduce recalcitrance. Similarly, pectin degradation in crop plants as well as in energy plants can enhance saccharification and enable the remaining polymers to be better substrates for the production of biofuels [43-45]. The genetic manipulation of xylan and pectin biosynthetic genes is a potential means to develop Populus plants with reduced xylan and pectin content and biomass that yields increased sugar release for biofuel production.

Here, we report that targeted RNAi constructdriven downregulation of one of the Populus deltoides GAUT12 orthologs (POPTR_0001s44250/ Potri.001G416800, GAUT12.1-KD) results in plants with reduced pectin and xylan content; increased plant height, stem expansion, and biomass yield; and greater glucose and xylose release and lignin syringyl-to-guaiacyl (S/G) ratio. No change in the amount of total lignin was observed in these transgenic lines nor was an irregular xylem cell phenotype observed in secondary Populus wood as has been reported in the Arabidopsis irx 8 mutant. There was, however, a significant increase in phloem fiber cell length and width and in xylem fiber and vessel cell size in PdGAUT12.1 knockdown (PdGAUT12.1-KD) wood. PdGAUT12.1-KD lines also exhibited larger leaf area, larger palisade, and spongy parenchyma cell size and greater relative water content compared to controls.

\section{Results and discussion}

\section{GAUT12 is highly expressed in xylem in P. deltoides}

The GAUT1-related gene family consists of 15 GAUTs in Arabidopsis and 20 GAUTs in P. trichocarpa [46], suggesting that one or two poplar orthologs exist for each Arabidopsis GAUT gene. The Arabidopsis GAUT family falls into three broad clades in which clade $A$ is subdivided into clades A1-4, clade B into clades B 1-2, and clade $C$ is undivided [46]. A phylogenetic analysis of the 15 Arabidopsis GAUT proteins (TAIR10) and the 20 P. trichocarpa GAUTs (Phytozome 8.0) using MEGA5 [47] confirmed the family structure as previously described [46]. There are two Populus orthologs of Arabidopsis GAUT12 (IRX8) in clade C (Figure 1) [46]. For the purposes of this paper, we named them GAUT12.1 (encoded by POPTR_0001s44250, Genemodel V2.0, Phytozome 8.0; Potri.001G416800, Genemodel V3.0, Phytozome 10.0) and GAUT12.2 (encoded by POPTR_0011s13600, Genemodel V2.0, Phytozome 8.0; Potri.011G132600, Genemodel V3.0, Phytozome 10.0). PtGAUT12.1 has $82 \%$ amino acid identity (Figure 2A) and $75 \%$ nucleotide sequence identity to AtGAUT12. The two Populus GAUT12 proteins, PtGAUT12.1 and PtGAUT12.2, share 91\% amino acid sequence identity and $90 \%$ nucleotide sequence identity with each other.

Quantitative real-time (RT)-PCR was used to measure the transcript level of PdGAUT12.1 and PdGAUT12.2 in $P$. deltoides. Both genes were most highly expressed in differentiating xylem; however, PdGAUT12.1 expression was approximately eight times greater than PdGAUT12.2 (Figure 2B,C) [37,38]. PdGAUT12.1 transcript expression in leaf, stem, and petiole was lower than in stem cambium in $P$. deltoides (Figure $2 \mathrm{~B}$ ). No PdGAUT12.2 transcript was detected in leaf and there was low expression in the petiole and stem (Figure $2 \mathrm{C}$ ). Due to the abundant expression of PdGAUT12.1 transcript in differentiating Populus xylem, we characterized the effect of reduced expression of this gene on plant and wood development and sugar release. Several prior reports described aspects of the function of this gene during wood formation in Populus [37,39]. However, this is the first report of a knockdown (KD) targeted against the PdGAUT12.1 gene (PdGAUT12.1$\mathrm{KD})$ alone and of the resulting phenotypes.

\section{Downregulation of the expression of GAUT12.1 in $P$. deltoides by RNAi-silencing}

An RNAi construct (Figure 3B) containing PtGAUT12.1specific sequence to trigger RNAi-silencing of PdGAUT12.1, but not PdGAUT12.2 (Figure 3A), was generated to study the function of GAUT12 during wood development. The construct was transferred into eastern cottonwood ( $P$. deltoides) clone WV94. Integration of the PdGAUT12.1 RNAi construct into the 


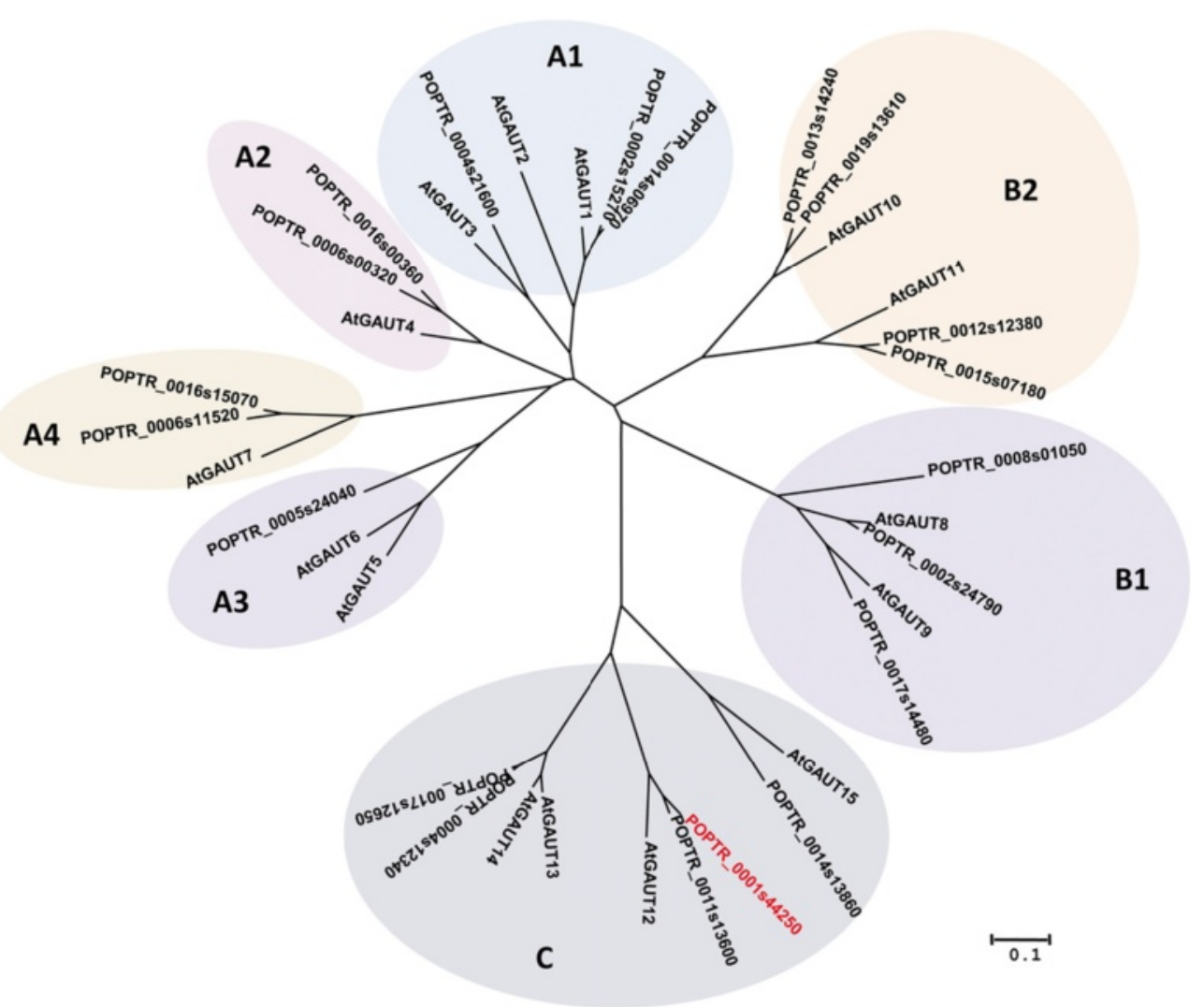

Figure 1 Phylogenetic tree of GAUTs in Arabidopsis thaliana and Populus trichocarpa. Phylogenetic tree showing the relationship between amino acid sequences of the GAUT Protein Family of Arabidopsis thaliana (TAIR10) and Populus trichocarpa (Phytozome 8.0). The tree was constructed by the Neighbor-Joining method using MEGA5 [44]. POPTR_0001s44250 is named GAUT12.1 in this paper.

genome was confirmed by gene-specific PCR of leaf DNA from the 11 PdGAUT12.1-KD transgenic lines obtained, AB30.1 through AB30.11 (data not shown). The WT nontransformed cottonwood ( $P$. deltoides) clone WV94 and eight plants transformed with the vector served as controls. Vector control lines were verified by PCR to contain the control construct (data not shown). Transgenic plants were produced by regeneration of shoots from transformed callus grown on shoot elongation medium, transfer of 3- to 5-cm-tall shoots from callus to BTM medium for rooting [48], and propagation of rooted seedlings to amplify the clonal lines. WT WV94 (25 plants), 10 to 15 clones of each vector control line, and all 11 PdGAUT12.1-KD transgenic lines were analyzed for this study.

Transcript expression of PdGAUT12.1 was measured by quantitative RT-PCR. The transcript level of PdGAUT12.1 was reduced by 50 to $67 \%$ in GAUT12.1$\mathrm{KD}$ lines compared to the controls (Figure 3C). Two patterns of GAUT12.1 transcript reduction were observed. Transgenic lines AB30.1, AB30.2, AB30.3, AB30.5, $\mathrm{AB} 30.8, \mathrm{AB} 30.10$, and $\mathrm{AB} 30.11$ had 60 to $67 \%$ reduced GAUT12.1 transcript levels and lines AB30.4, AB30.6, $\mathrm{AB} 30.7$, and $\mathrm{AB} 30.9$ were reduced by 50 to $54 \%$. We also quantified the transcript level of GAUT12.2 to determine whether the sequence-specific RNAi construct for PdGAUT12.1 had any effect on PdGAUT12.2 transcript expression in the lines. No significant change in PdGAUT12.2 transcript level was observed in any of the transgenic lines in relation to control plants (Figure 3C).

\section{RNAi downregulation of $P$. deltoides GAUT12.1 expression leads to improved saccharification without changing the amount of total lignin}

To determine the effect of downregulation of PdGAUT12.1 on the recalcitrance of Populus to enzymatic deconstruction, 9-month-old WT, vector control, and PdGAUT12.1-KD transgenic lines were subjected to pretreatment and enzymatic hydrolysis. Glucose release per gram dry biomass was significantly increased by 4 to 8\% across ten of the eleven PdGAUT12.1-KD lines in comparison to the controls (Figure 4A). Xylose release per gram dry biomass was increased 3 to $7 \%$ in ten of the eleven transgenic lines (Figure 4B). Total sugar release in all 11 PdGAUT12.1-KD lines increased 4 to $6 \%$ compared to WT and vector controls (Figure 4C). 


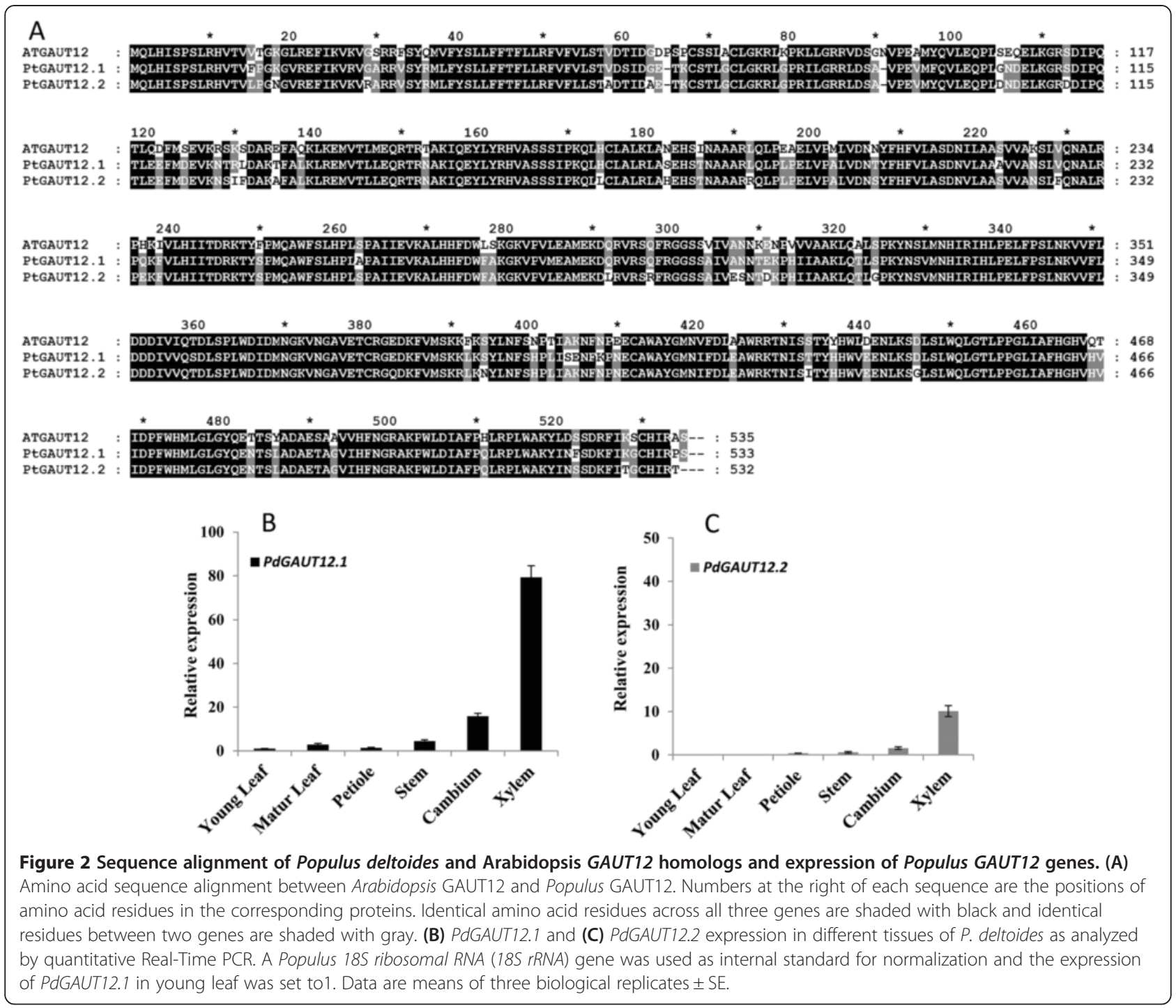

Arabidopsis irx8/gaut12 mutants have been reported to have reduced levels of lignin [30,49]. To determine if the reduced recalcitrance in the Populus PdGAUT12.1$\mathrm{KD}$ lines was associated with reduced lignin content, total lignin and lignin S/G levels were measured by pyrolysis molecular beam mass spectrometry. There was no significant difference in the total lignin content of the PdGAUT12.1-KD (26.25\% dry biomass weight) compared to WT (26.13\%) and vector controls (26.19\%) (Table 1). This is in contrast to the report on double RNAi-silenced PtrGT8D1 and PtGT8D2 P. trichocarpa lines which had 11 to $25 \%$ increased lignin content [37].

All 11 PdGAUT12.1-KD lines analyzed showed a significant increase in lignin $\mathrm{S} / \mathrm{G}$ ratio levels (Table 1), similar to the Arabidopsis irx8 mutant [30]. There was a positive correlation between $\mathrm{S} / \mathrm{G}$ ratio and glucose, xylose, and total sugar release $\left(R^{2} 0.77,0.81\right.$, and 0.81 , respectively). No correlation was observed between sugar release and total lignin content in PdGAUT12.1KD lines. The results indicate that knockdown expression of GAUT12.1 in P. deltoides results in increased sugar release and lignin $S / G$ ratios.

A small reduction in $\mathrm{S}$ lignin was previously reported in P. alba $x$ tremula PoGT8D RNAi lines [39]. However, in the present study with $P$. deltoides the $\mathrm{S} / \mathrm{G}$ ratio was significantly increased (3 to 7\%) in the PdGAUT12.1-KD lines compared to WT and vector control plants (Table 1). Total sugar release was previously shown to increase with increasing S/G ratio [50]. However, in that study, glucose and total sugar release were negatively correlated with lignin content. In the present study, although total lignin levels did not differ between the control and transgenic lines, there was still a positive correlation between high S/G ratios and increased sugar release in the PdGAUT12.1-KD lines, suggesting that GAUT12.1 expression affects the lignin composition in 


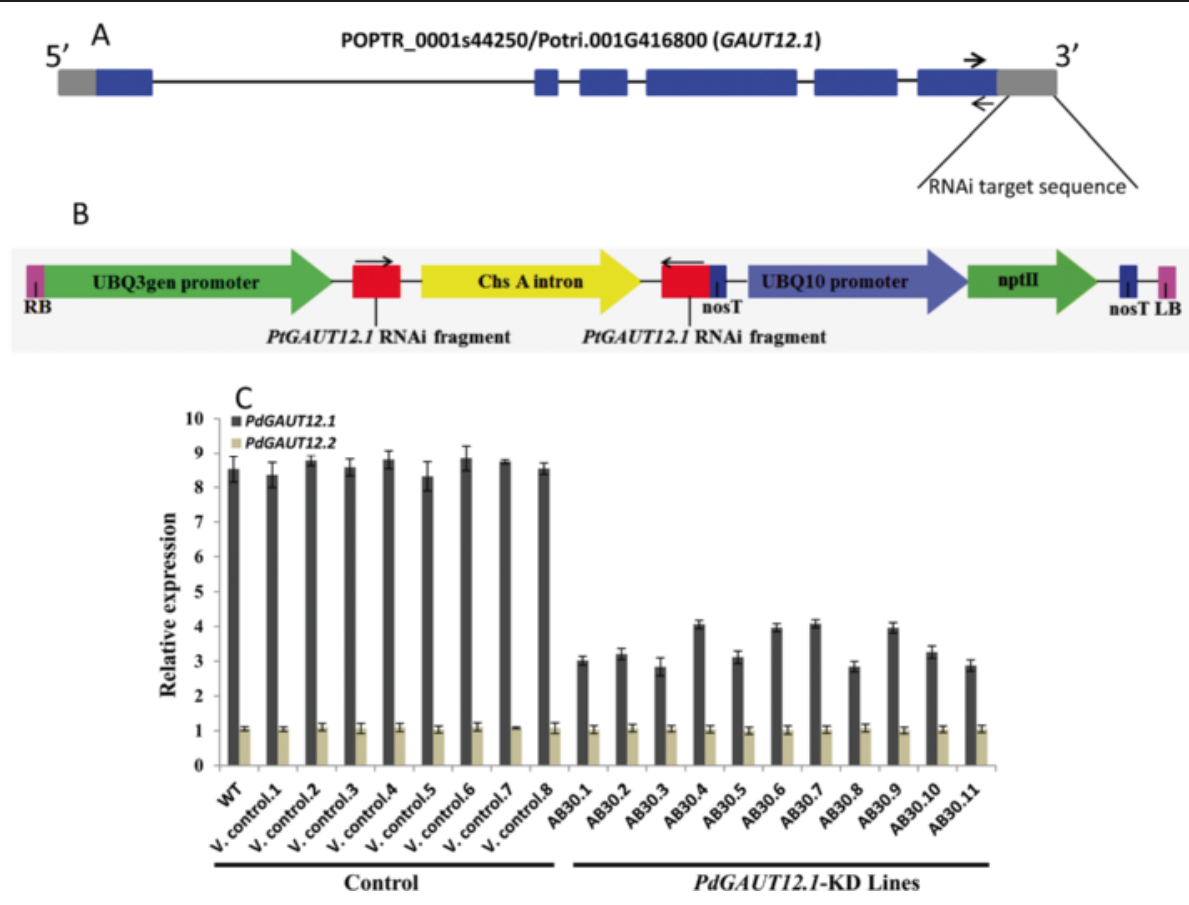

Figure 3 Knockdown of PdGAUT12.1 in Populus deltoides. (A) Positions of the RNAi target sequence in the GAUT12.1 (POPTR_0001544250/ Potri.001G416800) gene. Blue boxes indicate exons, lines indicate introns, and gray boxes are the 5' and 3' untranslated regions (UTRs). The RNAi target sequence was $168 \mathrm{bp}$ in the $3^{\prime} \mathrm{UTR}$. The arrows indicate the targeted sequences used for quantitative real-time PCR. (B) Schematic presentation of PdGAUT12.1 RNAi silencing construct. (C) Quantitative real-time PCR analysis of controls and PdGAUT12.1-KD lines of 3-month-old plants. The relative expression level of each gene was normalized using Populus 185 ribosomal RNA (18S rRNA) as the reference gene and the expression of PdGAUT12.2 in AB30.5 was set to 1. $N=5$. Error bars represent SE.

wood and that the composition of the lignin is a factor affecting recalcitrance. It is also noteworthy that, contrary to the results obtained here, simultaneous knockdown expression of both Populus GAUT12 homologs in P. trichocarpa resulted in 11 to $25 \%$ increased lignin content [37]. As there was no measurement of biomass recalcitrance in that report, it is not known how the transgenic plants were affected in sugar release. Other studies, however, have shown that increased lignin content is detrimental to effective sugar release [50-52]. The knockdown of both GAUT12 homologs in P. trichocarpa transgenics also resulted in more brittle wood, 17 to $29 \%$ reduced stem modulus of elasticity and 16 to $23 \%$ reduced modulus of rupture [37], suggesting that reduced expression of both homologs may be deleterious to wood quality.

\section{RNAi downregulation of PdGAUT12.1 results in increased plant growth in Poplar}

Substantial morphological differences were observed between the controls and PdGAUT12.1-KD transgenic lines. Measurement of plant height and stem diameter at 3 months showed that PdGAUT12.1 RNAi-silenced transgenic lines had increased vegetative growth compared to controls (Figure 5A). Nine of the eleven PdGAUT12.1-KD transgenic lines had 12 to 52\% increased plant height compared to WT and vector control plants (Table 2), and nine lines had 12 to $44 \%$ greater stem diameter (Table 2). We selected four transgenic lines (AB30.1, AB30.3, AB30.8, AB30.11) for further phenotype analysis based on GAUT12.1 transcript expression and growth phenotype, lignin S/G ratio, and the saccharification data. Measurement of plant height and stem diameter over a 9-month growth period showed that the increased height and stem diameter of the PdGAUT12.1-KD transgenic lines continued throughout the growth period (Figure 5B,C). Indeed, the total aerial biomass of the PdGAUT12.1-KD plants was $17 \%$ to $38 \%$ greater than controls (Figure 5D) by the end of the 9-month growth period. The increase in plant height and stem diameter were negatively correlated $\left(R^{2}\right.$ of 0.66 and 0.75 , respectively) with GAUT12.1 transcript levels (Additional file 1), which were reduced by 50 to $67 \%$ compared to WT and vector control plants. These results suggest that systematic manipulation of the GAUT12.1 gene can lead to greater plant growth.

The PdGAUT12.1-KD plants had increased vegetative growth compared to controls. This is in contrast to Arabidopsis ir $x 8$ mutant plants which have stunted growth $[8,10,29]$. This difference may be due to the 50 to $67 \%$ reduction in GAUT12.1 transcript level in the RNAi- 

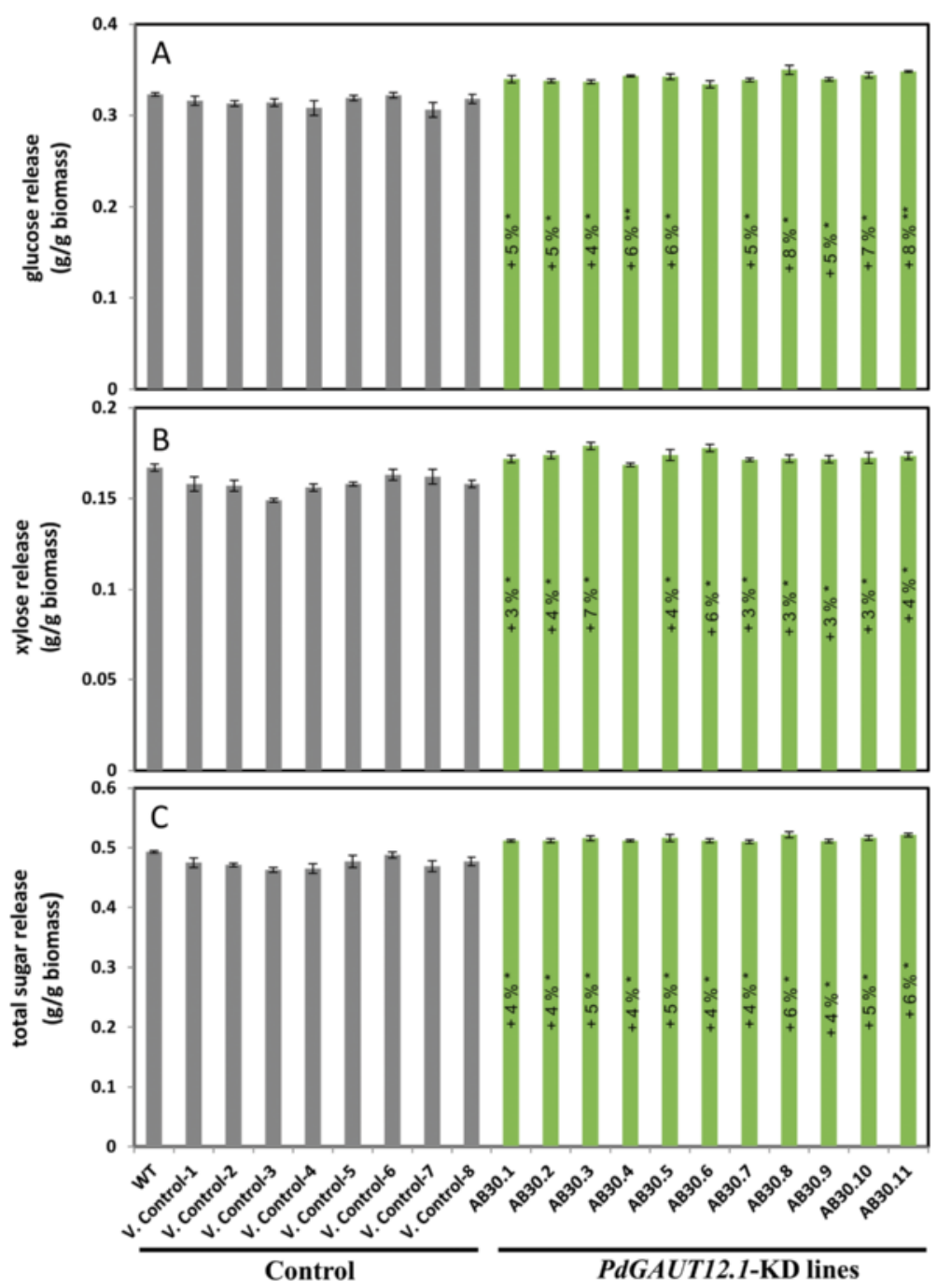

Figure 4 RNAi downregulation of $P$. delotides PdGAUT12.1 expression leads to improved saccharification. Glucose (A), xylose (B) and total sugar (C) release from controls and PdGAUT12.1-KD lines. Significance $P$ values are expressed as ${ }^{*} P<0.05,{ }^{*} P<0.001$ calculated by statistical analysis using Statistica 5.0 with one-way analysis of variance (ANOVA) followed by Tukey's multiple comparison test. $n=25$ for wild-type $P$. deltoides, $n=10$ to 15 for vector control and PdGAUT12.1-KD lines.

silenced $P$. deltoides transgenic plants compared to the total knock out of GAUT12/IRX8 in the Arabidopsis irx8 mutants [8,29]. However, the previously reported simultaneous knockdown expression of both PtrGT8D1 and PtGT8D2 in P. trichocarpa, similar to the work described here, also did not yield a dwarf phenotype in Populus even though those plants had 85 to $94 \%$ reduced GAUT12 transcript levels [37]. The PtrGT8D RNAi-silenced transgenic plants [37] also did not have collapsed vessels as observed in the Arabidopsis irx 8 mutant $[8,29]$. Based on those results, it was proposed that growth of the PtrGT8D RNAi-silenced transgenic plants was not affected because the vessels in the transgenic lines retained their normal transport function [37]. The simultaneous knockdown expression of both
Populus GAUT12 homologs in P. trichocarpa [33], however, did not result in the increased growth observed in the current study where only GAUT12.1 expression was reduced. The increased plant size obtained upon targeted downregulation of a specific GAUT12 homolog as reported here suggests that a controlled manipulation of this gene to yield greater plant growth could be used to improve feedstock for the biofuel industry.

Altered PdGAUT12.1 expression in leaves causes increased leaf cell size and number and increased relative water content

To determine how leaf growth and size was affected in the PdGAUT12.1-KD lines, every fifth successive leaf from the apex down to leaf 40 (Additional file 2C-D) 
Table 1 Total lignin content and S/G ratio of controls and PdGAUT12.1-KD lines

\begin{tabular}{lll}
\hline Plant types & Lignin content (\%) & S/G ratio \\
\hline WT & $26.13 \pm 0.15$ & $1.90 \pm 0.02$ \\
V Control-1 & $25.82 \pm 0.17$ & $1.68 \pm 0.01$ \\
V Control-2 & $26.68 \pm 0.35$ & $1.70 \pm 0.03$ \\
V Control-3 & $26.00 \pm 0.34$ & $1.62 \pm 0.02$ \\
V Control-4 & $26.45 \pm 0.21$ & $1.73 \pm 0.03$ \\
V Control-5 & $26.43 \pm 0.42$ & $1.85 \pm 0.03$ \\
V Control-6 & $26.24 \pm 0.16$ & $1.85 \pm 0.04$ \\
V Control-7 & $25.61 \pm 0.40$ & $1.62 \pm 0.07$ \\
V Control-8 & $26.30 \pm 0.25$ & $1.78 \pm 0.05$ \\
AB30.1 & $25.53 \pm 0.50$ & $2.00 \pm 0.03^{*}$ \\
AB30.2 & $25.99 \pm 0.39$ & $1.96 \pm 0.02^{*}$ \\
AB30.3 & $26.37 \pm 0.15$ & $1.98 \pm 0.02 *$ \\
AB30.4 & $26.61 \pm 0.26$ & $1.98 \pm 0.03^{*}$ \\
AB30.5 & $26.84 \pm 0.21$ & $1.97 \pm 0.03^{*}$ \\
AB30.6 & $26.11 \pm 0.41$ & $2.02 \pm 0.04^{*}$ \\
AB30.7 & $26.43 \pm 0.38$ & $1.97 \pm 0.02^{*}$ \\
AB30.8 & $25.78 \pm 0.37$ & $1.97 \pm 0.02^{*}$ \\
AB30.9 & $26.44 \pm 0.63$ & $2.04 \pm 0.02^{*}$ \\
AB30.10 & $26.38 \pm 0.24$ & $1.98 \pm 0.02^{*}$ \\
AB30.11 & $26.28 \pm 0.17$ & $2.03 \pm 0.02^{*}$ \\
\hline
\end{tabular}

Lignin content is expressed as percentage (wt/wt). S/G ratios were determined by summing the intensity of the syringyl peaks at 154, 167, 168, 182, 194, 208, and 210 and dividing by the sum of intensity of guaiacyl peaks $124,137,138$, 150,164 , and 178 (see methods for details, Additional file 9). Mean $\pm S E$, Significant $P$ values are expressed as ${ }^{*} P<0.05,{ }^{*} P<0.001$ as calculated by statistical analysis using Statistica 5.0 with one-way analysis of variance (ANOVA) followed by Tukey's multiple comparison test. $n=25$ for wild-type $P$. deltoides, $n=10$ to 15 for vector control (v control-1 to v control-8) and PdGAUT12.1-KD lines (AB30.1-AB30.11).

was examined. Both leaf length and width was significantly $(P=0.05)$ larger for each of the PdGAUT12.1-KD lines examined compared to the controls (Additional file 2C-D). To compare the growth of developing leaves, every fifth, sixth, and seventh leaf from the apex was collected (Additional file 2B, E) and leaf area was measured. All PdGAUT12.1-KD lines examined had 52 to $117 \%$ significantly greater leaf area compared to control plants (Additional file 2E). Simultaneously, we collected the 20th to 40th leaf from the apex of five transgenic and control lines and compared the fully expanded leaf area. All four transgenic lines had 22 to $76 \%$ greater leaf area than control plants (Additional file $2 \mathrm{~F}$ ). Therefore, both the leaf length and width was significantly greater in both the developing and expanded leaves of PdGAUT12.1-KD lines resulting in a larger leaf area. The PdGAUT12.1-KD lines also had accelerated growth in the top part of the PdGAUT12.1-KD plant (Additional file $2 \mathrm{~A}$ ) and 2.8 times greater biomass yield compared to WT (Additional file 2H).
To establish whether the larger leaf size in the PdGAUT12.1-KD lines was a result of increased cell number or cell expansion, we analyzed cross sections of leaf blades of the sixth leaf from the apex of the plant and hand cut cross sections of the 20th leaf. The knockdown lines had increased numbers of palisade parenchyma cells $(17 \%, \mathrm{AB} 30.1 ; 13 \%, \mathrm{AB} 30.8)$ compared to WT (Additional file 3A-C, M). The knockdown lines also had larger bundle-sheath cells (Additional file 3DG, I, K). Although there were more palisade cells in the young leaves, by the time these leaves matured, there was no overall major change in cross sectional total leaf area occupied by palisade cells of PdGAUT12.1-KD lines compared to WT, except for a small $9 \%$ increase in line AB30.1 (Additional file 4A-F). As shown in Additional file 2; however, there was a significant increase in the total leaf surface area in the mature PdGAUT12.1-KD leaves compared to the control (Additional file 2C-F). Also, the palisade and spongy parenchyma cells in mature leaves of PdGAUT12.1-KD lines were 13 to 56\% and 23 to $62 \%$ larger, respectively, than in the controls (Additional file 4G-H). Thus, the increased leaf size of the PdGAUT12.1-KD lines is due to both increased cell number and cell size.

Leaf water content is a useful indicator of plant water balance and expresses the relative amount of water present in the plant tissues. The measurement of relative water content (RWC) in leaf tissues is commonly used to assess the water status of plants. Measurements of water content in a tissue on a fresh or dry mass basis can be correlated with the maximum amount of water a tissue can hold. Leaf water status is positively correlated with several leaf physiological variables, such as leaf turgor, transpiration, stomatal conductance, and growth [53]. We thus measured the RWC of PdGAUT12.1-KD lines to determine if it was correlated with the positive growth effect observed in the lines. All four knockdown lines examined had 5 to $10 \%$ increased RWC compared to the WT at $72 \mathrm{~h}$ (Additional file 2G). After $72 \mathrm{~h}$, there was a 7\% increase in RWC of PdGAUT12.1-KD plants compared to the WT (Additional file 2G). In summary, the results show that the increased leaf size in the PdGAUT12.1-KD lines could be explained by enhanced cell number, enhanced cell size, and enhanced RWC.

\section{Downregulation of PdGAUT12.1 in secondary cell wall-containing tissues leads to increased phloem and vessel size}

To examine the role of downregulation of GAUT12.1 in tissues containing secondary walls, stems from transgenic lines and WT were analyzed by sectioning internode numbers 10 and 20 from the top of the stem. A comparison of the internode 10 stem cross sections from PdGAUT12.1-KD lines versus WT revealed a significant 

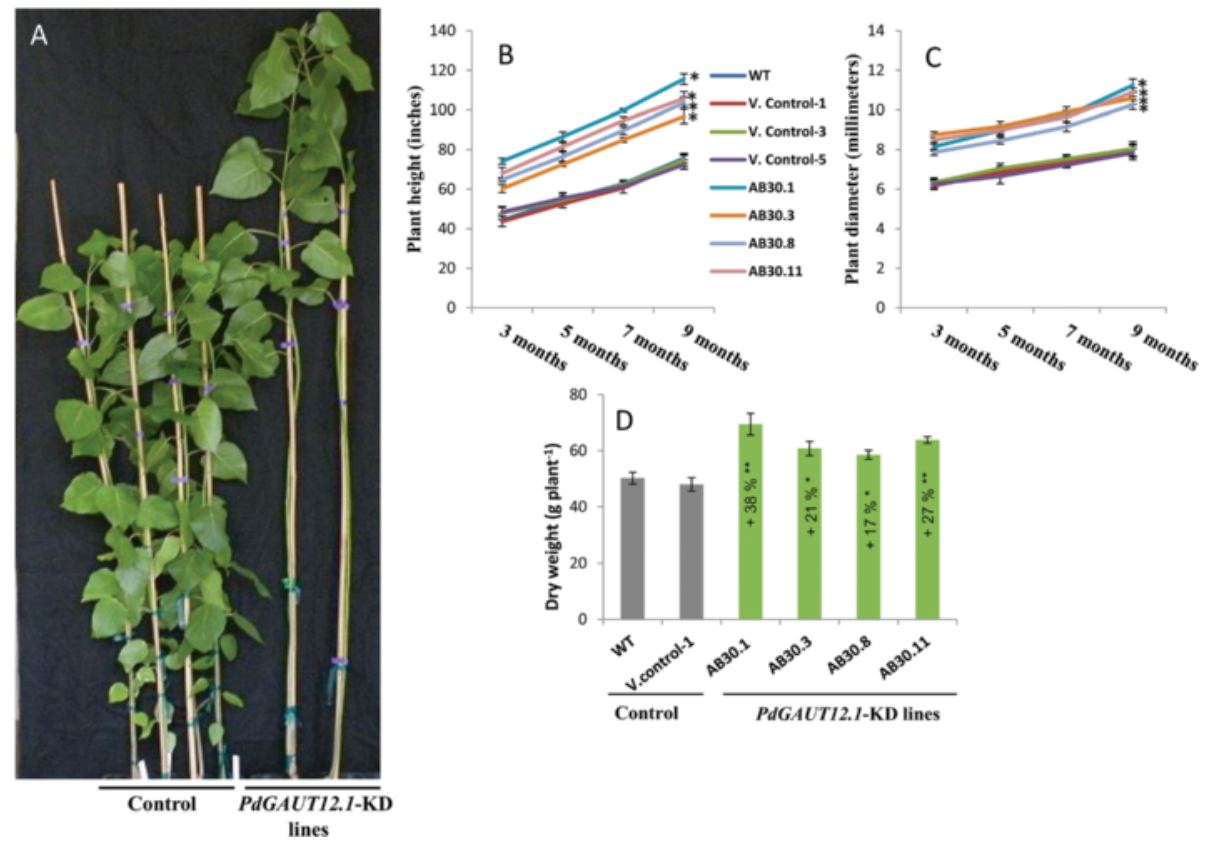

Figure 5 RNAi downregulation of PdGAUT12.1 results in increased plant growth. Phenotype and development of PdGAUT12.1-KD transgenic lines. (A) Plant phenotypes of 3-month-old wild-type P. deltoides (left two plants), vector control (middle two plants), and PdGAUT12.1-KD lines (right two plants). (B) Height and (C) radial growth of PdGAUT12.1-KD lines in comparison to the controls. (D) Dry aerial biomass of WT and PdGAUT12.1-KD lines of three-month-old plants. $n=7$ for plant height and stem diameter, $n=6$ for dry weight measurement. Asterisks indicate values significantly different from WT by ANOVA followed by Tukey's multiple comparison test, ${ }^{*} P<0.05$, ${ }^{* *} P<0.001$. Error bars represent SE.

increase in the PdGAUT12.1-KD lines of the amount of phloem tissue $(63 \%, \mathrm{AB} 30.1 ; 69 \%$, AB30.8) compared to WT (Figure 6A,B,D,E,G,H,S). We did not observe the collapsed xylem cell phenotype reported for the irx 8 Arabidopsis mutants [8,29]. However, there was a significant increase in xylem vessel cell size in the PdGAUT12.1-KD lines compared to WT. The 10th internode from the transgenic lines had $40 \%$ and $31 \%$ larger vessel diameter than WT (Figure 6A,C,D,F,G,I,T). Our data corroborate similar vessel phenotypes previously reported in P. trichocarpa when both PtGAUT12.1 and PtGAUT12.2 were simultaneously knocked down through RNAi silencing [37]. We also observed an increased amount of phloem (50\%, AB30.1; 55\%, AB30.8) and increased xylem vessel cell lumen diameter (48\%, AB30.1; $39 \%, \mathrm{AB} 30.8)$ in the 20th internodes from the transgenic lines compared to the WT (Figure 6J to R,U,V).

Since we observed increased stem diameter in the PdGAUT12.1-KD lines, we hypothesized that increased wood diameter was a result of an increased radial expansion of the xylem cells. To test this hypothesis, wood cells from internode 40 of the transgenic and WT plants were separated by maceration. The measurement of fiber cell size revealed that all knockdown lines had greater fiber cell length $(19 \%$ to $26 \%)$ and two transgenic lines had wider fiber cells $(6 \%, 8 \%)$ than the WT (Figure 7E,F). Vessel cell length and diameter were also measured in the WT and
PdGAUT12.1-KD lines (Figure 7D). All transgenic lines examined had significantly larger vessel cell total length $(12 \%$ to $21 \%$ ) and vessel cell lumen length (7\% to $9 \%$ ) and wider vessel cell diameter $(12 \%$ to $29 \%$ ) than WT vessels (Figure 7A to C). Thus, at least a portion of the change in stem length and diameter in PdGAUT12.1-KD lines is likely due to increased xylem and vessel cell size. The larger vessel size may also support the larger leaf size and greater water content of the pdGAUT12-KD lines.

\section{Populus PdGAUT12.1 complements and PdGAUT12.2} partially complements the Arabidopsis irx8 (gaut12) mutant To examine whether PdGAUT12.1 and PdGAUT12.2 are functional homologs of the Arabidopsis GAUT12, we transformed T-DNA constructs harboring the full-length coding sequences of Arabidopsis thaliana GAUT12 as well as $P$. deltoides GAUT12.1 and GAUT12.2 (Additional file 5) under the control of a CaMV $35 \mathrm{~S}$ promoter into irx8-5/+ plants. For each construct, approximately 12 primary transgenics homozygous for the irx 8 allele were selected and their phenotypes examined. Both the AtGAUT12 and PdGAUT12.1 constructs showed similar activity in complementing irx8: about $20 \%$ of the primary transgenics did not show any of the visible defects associated with the mutation; these plants produced WT-like rosette leaves and intact vessels (Figure 8A to $\mathrm{C}$ ), resulting in significantly increased rosette size and overall height (Figure 8D,E). In 


\begin{tabular}{|c|c|c|}
\hline Plant types & Plant height (inches) & Plant diameter $(\mathrm{mm})$ \\
\hline WT & $48.31 \pm 3.1$ & $6.3 \pm 0.24$ \\
\hline V Control-1 & $46.67 \pm 2.5$ & $6.1 \pm 0.19$ \\
\hline V Control-2 & $47.18 \pm 0.9$ & $6.2 \pm 0.13$ \\
\hline V Control-3 & $47.95 \pm 1.2$ & $6.3 \pm 0.26$ \\
\hline V Control-4 & $47.62 \pm 1.9$ & $6.1 \pm 0.31$ \\
\hline V Control-5 & $48.43 \pm 2.0$ & $6.3 \pm 0.37$ \\
\hline V Control-6 & $48.51 \pm 1.5$ & $6.1 \pm 0.36$ \\
\hline V Control-7 & $48.81 \pm 2.0$ & $6.3 \pm 0.22$ \\
\hline V Control-8 & $48.81 \pm 1.0$ & $6.1 \pm 0.23$ \\
\hline AB30.1 & $74.17 \pm 2.9^{* *}$ & $8.2 \pm 0.46^{* *}$ \\
\hline AB30.2 & $61.81 \pm 3.3^{* *}$ & $7.7 \pm 0.34^{* *}$ \\
\hline AB30.3 & $60.29 \pm 2.3^{* *}$ & $8.7 \pm 0.32^{* *}$ \\
\hline AB30.4 & $49.22 \pm 2.2$ & $6.8 \pm 0.29$ \\
\hline AB30.5 & $60.86 \pm 2.0^{* *}$ & $9.1 \pm 0.53^{* *}$ \\
\hline AB30.6 & $54.61 \pm 3.8^{* *}$ & $6.6 \pm 0.41$ \\
\hline AB30.7 & $49.86 \pm 2.4$ & $7.1 \pm 0.28^{* *}$ \\
\hline AB30.8 & $64.75 \pm 2.4^{* *}$ & $7.9 \pm 0.37^{* *}$ \\
\hline AB30.9 & $54.82 \pm 1.0^{* *}$ & $7.2 \pm 0.37^{* *}$ \\
\hline AB30.10 & $61.72 \pm 2.3^{* *}$ & $8.4 \pm 0.30^{* *}$ \\
\hline AB30.11 & $68.07 \pm 2.0^{* *}$ & $8.5 \pm 0.33^{* *}$ \\
\hline
\end{tabular}

Plant height and plant diameter of three-month-old wild type $P$. deltoides, vector control and $P d G A U T 12.1-\mathrm{KD}$ lines. Means $\pm \mathrm{SE}$, Significance $P$ values are expressed as ${ }^{* *} P<0.05,{ }^{* *} P<0.001$ as calculated by statistical analysis using Statistica 5.0 with one-way analysis of variance (ANOVA) followed by Tukey's multiple comparison test. $\mathrm{n}=25$ for wild type $P$. deltoides, $\mathrm{n}=10$ to 15 for vector control ( $\mathrm{v}$ control- 1 to $v$ control-8) and PdGAUT12.1-KD lines (AB30.1-AB30.11).

contrast, the PdGAUT12.2 construct only partially rescued the phenotypes associated with $i r x 8$, as primary transgenics had only marginally larger rosette leaves and slightly taller stature (Figure 8D,E). The PdGAUT12.2 lines displaying the strongest rescue effect still had many collapsed xylem vessels (Figure 8C). We conclude that the Poplar PdGAUT12.1 gene is as effective in complementing Arabidopsis irx 8 mutants as the native GAUT12 gene.

\section{RNAi downregulation of GAUT12.1 expression leads to reductions in pectin and xylan content and increased extractability of $P$. deltoides wood \\ Sugar composition analyses of total cell walls from PdGAUT12.1-KD plants}

We investigated the effects of RNAi downregulation of $P$. deltoides GAUT12.1 expression on the content of cell wall polysaccharides in $P$. deltoides wood. Cell walls were isolated as alcohol-insoluble residues (AIRs) from cored, debarked wood from the bottom $6 \mathrm{~cm}$ of stems. All four PdGAUT12.1-KD lines had 17 to 30\% significantly reduced xylose content in AIR compared to control lines (Table 3), indicating that RNAi downregulation of $P$. deltoides
GAUT12.1 expression causes a defect in xylan formation. This result corroborated two earlier findings in Populus that PtGAUT12.1 has a role in xylan formation [37,39]. Simultaneously, the galacturonic acid (GalA) content was significantly reduced by $25 \%$ to $47 \%$ in all four transgenic lines compared to the WT. This result indicates that PdGAUT12.1-KD lines have defects in pectin formation in addition to the defect in xylan formation. A substantial increase in galactose and mannose content was also observed in PdGAUT12.1-KD walls compared to controls. The AIR composition data indicated a clear alteration in the composition of the PdGAUT12.1-KD walls compared to controls and indicated that at least two types of polymers, xylan and pectin, were affected.

\section{Glycosyl residue composition analyses of fractionated cell walls from PdGAUT12.1-KD plants}

The AIR (that is, cell walls) of control and transgenic lines were subjected to sequential extraction with increasingly harsh solvents to fractionate the walls to determine if cell wall changes caused by the GAUT12.1-KD could be identified in a specific subfraction of cell wall polymers. The total amount of material recovered in each sequential extract of the starting AIR for each of the six solvents was greater for the PdGAUT12.1-KD RNAi lines than for the controls. There was a significant 9 to $21 \%$ increase in the amount of material recovered in the ammonium oxalate extract, 7 to $15 \%$ increase in the carbonate extract, 17 to $34 \%$ increase in the $1 \mathrm{M} \mathrm{KOH}$ extract, 12 to $30 \%$ increase in the $4 \mathrm{M} \mathrm{KOH}$ extract, 8 to $10 \%$ increase in the chlorite extract, and 7 to $15 \%$ increase in the post-chlorite $4 \mathrm{M} \mathrm{KOH}$ extract, yielding an 11 to $17 \%$ overall increase in the total amount of extractable material from the starting AIR of the PdGAUT12.1-KD lines compared to WT (Additional file 6A-H). Since the total amount of AIR isolated from the WT versus the transgenic lines was comparable (Additional file 6A), these results suggested that the remaining insoluble wall material should have been reduced in the transgenic lines. Indeed, analysis of the mass of the final AIR pellet remaining after all extractions of AIR from the transgenic lines revealed that there was 6 to 13\% less final pellet from the PdGAUT12.1$\mathrm{KD}$ lines compared to control lines (Additional file 6I). These results suggest that GAUT12 functions in the synthesis of a structure that is highly integrated into the basic cell wall architecture, and that when GAUT12 function is reduced, the plants synthesize more easily extractable walls.

To determine if there were any differences in the type or mol\% amounts of each type of sugar in the different extracts from the PdGAUT12.1-KD lines compared to controls, the individual extracts were analyzed by glycosyl residue composition. Wall fractions extracted with mild reagents such as ammonium oxalate and sodium carbonate generally contain large amounts of GalA along with rhamnose (Rha), arabinose (Ara), galactose (Gal), glucose (Glc), xylose (Xyl), 


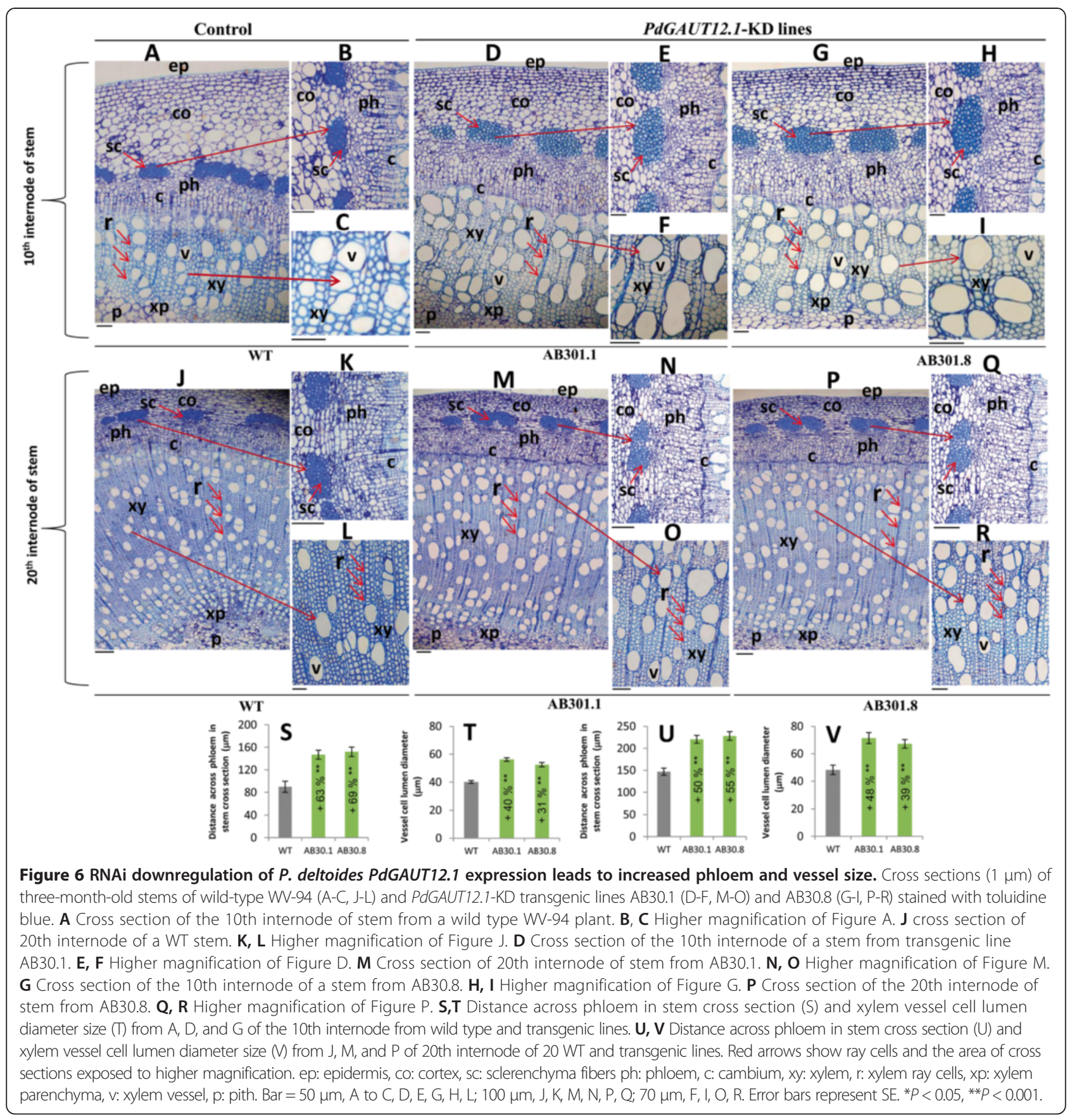

and mannose (Man) and, thus, are considered to be enriched in pectin. The oxalate extracts of AIR from the PdGAUT12.1-KD RNAi line had 39 to 78\% decreased mol $\%$ GalA compared to the controls, and the carbonate extracts had 17 to $42 \%$ decreased mol\% GalA (Table 4). The mol\% rhamnose in both the oxalate and carbonate fractions was also significantly reduced by 44 to $76 \%$ and 39 to $55 \%$, respectively, in the PdGAUT12.1-KD lines compared to the controls (Table 4). These results show that GAUT12.1 downregulation results in reduced amounts of easily extractable HG and RG. Simultaneously, the xylose, mannose, galactose, and glucose contents in the oxalate extracts were significantly increased in the PdGAUT12.1-KD lines compared to the WT (Table 4), while in the carbonate fractions, the glucose content was increased, suggesting that reduced levels of easily extractable pectin were accompanied by increased mol\% hemicellulose in these wall fractions.

As expected, extraction of the remaining insoluble wall residues with harsher alkaline solvents such as $1 \mathrm{M}$ and $4 \mathrm{M} \mathrm{KOH}$ (conditions which typically enrich for hemicelluloses) released primarily xylose with lesser amounts of glucose, mannose, GlcA, and galactose as well as 

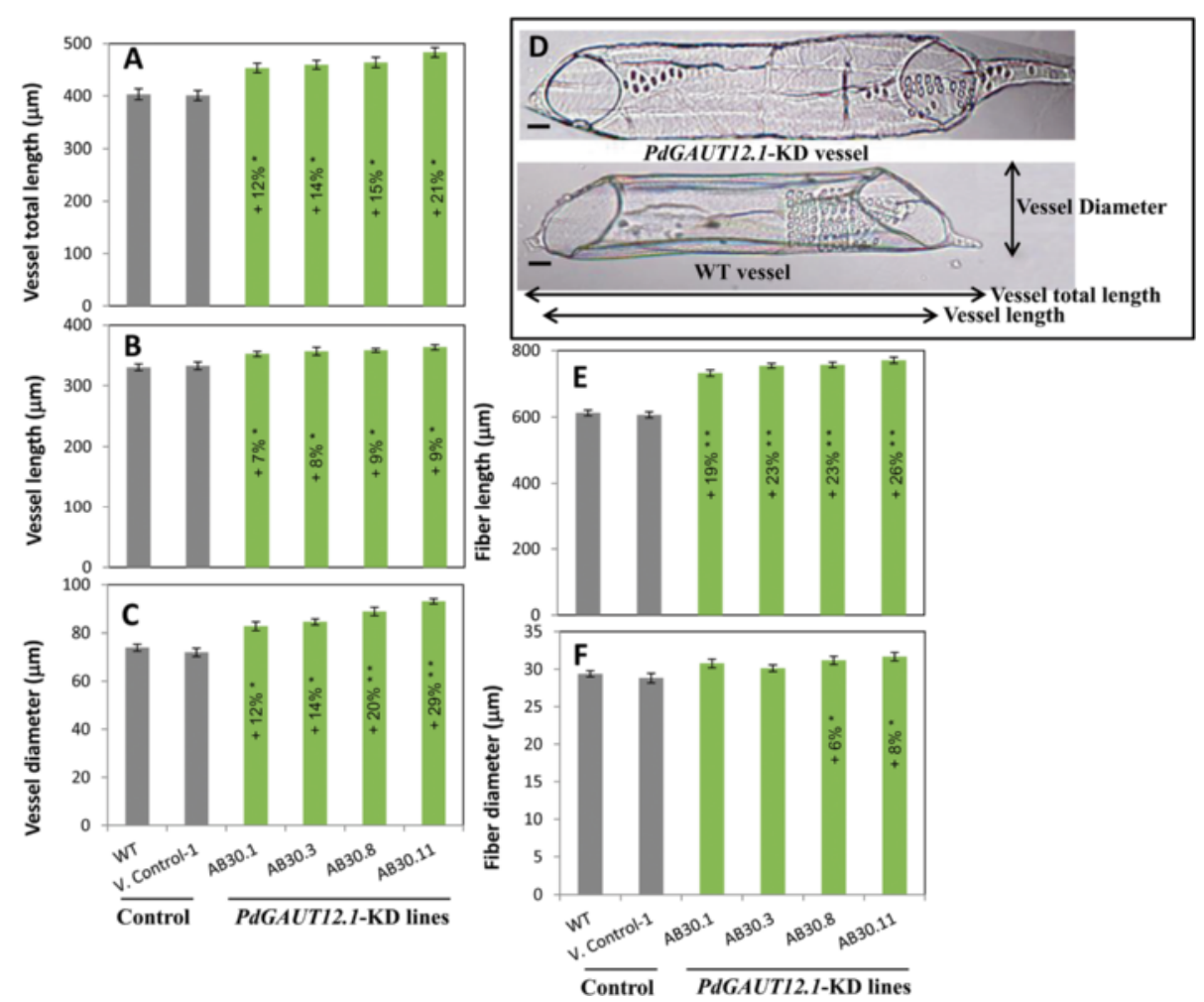

Figure 7 Xylem (fiber and vessel) cell size in WT and PdGAUT12.1-KD line. Downregulation of expression of GAUT12.1 results in increased xylem total vessel length (A), xylem lumen length (B), xylem vessel lumen diameter (C), xylem fiber cell length (E), xylem fiber cell diameter (F). Both xylem fibers and vessels were separated by maceration and approximately 80 fiber cells and 60 vessel elements from three independent PdGAUT12.1-KD transgenic lines were analyzed. Images were captured with a Nikon DS-Ri1 camera (Nikon, Melville, NY) using NIS-Elements Basic Research software. (D) Representative individual vessels of WT and PdGAUT12.1-KD lines illustrating the parameters measured. Asterisks indicate values significantly different from wild type based on ANOVA followed by Tukey's multiple comparison test, ${ }^{*} P<0.05,{ }^{*} P<0.001 . n=240$ fibers and 180 vessels cells. The vertical bar represents standard error. Bar $=20 \mu \mathrm{m}, \mathrm{D}$.

small amounts of rhamnose, GalA, and arabinonse. The PdGAUT12.1-KD $1 \mathrm{M} \mathrm{KOH}$ extract contained significantly reduced (35 to $45 \%)$ mol\% xylose compared to the controls (Table 4). There was also a 35 to $45 \%$ reduction in the mol\% GlcA and a 60 to $76 \%$ reduction in the mol\% GalA in these extracts from the PdGAUT12.1-KD lines. Similarly, we found a 31 to $41 \%$ reduction in xylose in the 4. M KOH extracts from PdGAUT12.1-KD RNAi lines. There was also a noticeable increase in mannose, galactose and glucose content in both the $1 \mathrm{M}$ and $4 \mathrm{M} \mathrm{KOH}$ extracts. The reduced 35 to $45 \%$ mol\% xylose and GlcA in the transgenic Populus plants (Table 4) agrees with the reduced xylan content in Arabidopsis irx8/gaut12 mutant plants $[8,29]$. The even larger reduction $(60$ to $76 \%)$ of GalA in the $1 \mathrm{M} \mathrm{KOH}$ extracts of the PdGAUT12.1-KD RNAi Populus lines suggests that reduced expression of this GAUT12.1 homolog may also impact a type of pectin that is released from the walls by this extraction.

More tightly bound lignin-associated components in the AIR can be removed by extraction with $100 \mathrm{mM}$ sodium chlorite. There was a 34 to $47 \%$ and 24 to $38 \%$ reduction of xylose and GalA, respectively, in the chlorite extract from the PdGAUT12.1-KD lines compared to the WT controls (Table 4). Similarly, a substantial decrease in arabinose (23 to 39\%) and rhamnose (14 to $37 \%$ ) was observed in this extract from the transgenic lines compared with controls.

After removal of lignin, the residual pellet following the chlorite extractions was treated with a second treatment of $4 \mathrm{M} \mathrm{KOH}$ to solubilize additional carbohydrates. Treatment with $4 \mathrm{M} \mathrm{KOH}$ after chlorite released primarily xylose. There was a 27 to $45 \%$ reduction in the amount of xylose in the post chlorite $4 \mathrm{M} \mathrm{KOH}$ extracts from PdGAUT12.1-KD lines compared to the controls (Table 4). Similarly, the post chlorite $4 \mathrm{M} \mathrm{KOH}$ extract from the PdGAUT12.1-KD lines had 27 to $45 \%$ reduced amounts of GlcA. No major change in GalA content was observed in this fraction (Table 4).

Taken together, the results indicate that downregulation of GAUT12.1 in Populus wood leads to reductions in a population of pectin and xylan. To compare the mass yield of each sugar from the starting AIR in the PdGAUT12.1-KD versus WT cell walls, the data were analyzed as micrograms sugar/milligram AIR. Analyses 


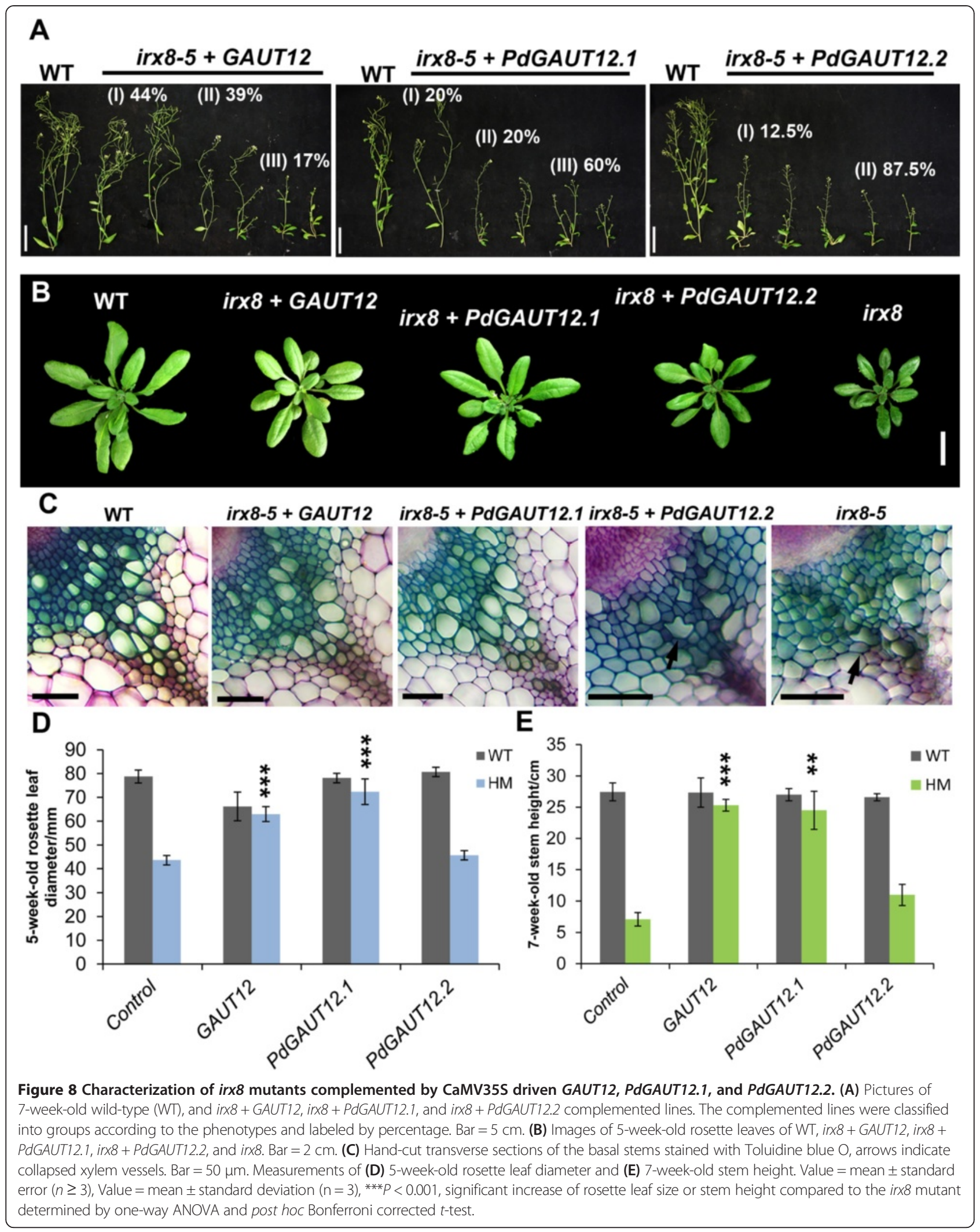


Table 3 Monosaccharide sugar composition of total cell walls (AIR) from nine-month-old Populus plants

\begin{tabular}{|c|c|c|c|c|c|c|c|c|c|}
\hline & \multicolumn{9}{|c|}{ Sugar composition of wood AIR stems walls (mol\%; \pm SE standard error) } \\
\hline & Ara & Rha & Fuc & Xyl & GlcA & GalA & Man & Gal & Glc \\
\hline WT & $3.11 \pm 0.11$ & $2.01 \pm 0.05$ & $0.08 \pm 0.01$ & $46.27 \pm 1.02$ & $0.23 \pm 0.01$ & $6.31 \pm 0.13$ & $6.29 \pm 0.41$ & $3.07 \pm 0.32$ & $33.21 \pm 0.85$ \\
\hline V Control-1 & $00 \pm 0.12$ & $1.87 \pm 0.1$ & 00 & 47.0 & 2 & 6.35 & 6.61 & 0.47 & 99 \\
\hline AB30.1 & $3.01 \pm 0.09$ & $2.31 \pm 0.12$ & $0.10 \pm 0.01$ & $38.33 \pm 0.56^{*}$ & $0.21 \pm 0.02$ & $4.70 \pm 0.14^{*}$ & $10.09 \pm 0.36^{*}$ & $7.32 \pm 0.33^{*}$ & $33.82 \pm 0.76$ \\
\hline AB30.3 & $2.95 \pm 0.08$ & $2.01 \pm 0.13$ & $0.06 \pm 0.01$ & $36.14 \pm 1.01^{*}$ & $0.21 \pm 0.01$ & $4.30 \pm 0.09^{*}$ & $11.58 \pm 0.38^{*}$ & $8.53 \pm 0.53^{*}$ & $34.19 \pm 1$ \\
\hline AB30.8 & $2.76 \pm 0.01$ & $1.76 \pm 0.21$ & $0.04 \pm 0.01$ & $32.30 \pm 0.85^{* *}$ & $0.20 \pm 0.01$ & $3.34 \pm 0.08^{* *}$ & $14.59 \pm 0.53^{*}$ & $10.99 \pm 0.58^{*}$ & $33.98 \pm 0.89$ \\
\hline AB30.11 & $2.86 \pm 0.08$ & $1.85 \pm 0.07$ & $0.17 \pm 0.02$ & $34.44 \pm 1.12^{* *}$ & $0.19 \pm 0.01$ & $3.79 \pm 0.11^{* *}$ & $11.72 \pm 0.39^{*}$ & $10.95 \pm 0.61^{*}$ & $33.96 \pm 0.97$ \\
\hline
\end{tabular}

Glycosyl residue composition of stem AIR as measured by GC-MS of tetramethylsilane (TMS) derivatives from controls and PdGAUT12.1-KD lines. The amount of sugar is represented as average mol\% of AIR (alcohol insoluble residue) isolated from stem walls. Means \pm SE of three biological replicates with two technical replicates each. Ital values with asterisks represent significant difference between WT and $P d G A U T 12.1-K D$ lines at ${ }^{*} P \leq 0.05$, ${ }^{* *} P \leq 0.001$ significant level (one-way ANOVA followed by Tukey's multiple comparison test).

of the total amount of each sugar in each extract of the starting cell wall (that is, AIR) (Additional file 7) show that only the GalA and rhamnose (Rha) were decreased in each PdGAUT12.1-KD cell wall extract compared to WT. These results could be consistent with HG and/or RG-I as the glycan directly affected in the PdGAUT12.1KD lines. However, significant reductions in Ara, Xyl, and GlcA were also observed, with the amount of Ara decreased in five of six extracts and Xyl and GlcA decreased in the four fractions released under the harshest extraction conditions, fractions known to be enriched for hemicelluloses. Thus, the data are also consistent with a role for GAUT12 in xylan synthesis.

\section{Glycosyl linkage analyses of fractionated cell walls from PdGAUT12.1-KD RNAi plants}

Glycosyl residue linkage analyses were carried out to test if the reductions in xylose, GalA, and Rha were associated with xylan, HG, and RG-I, respectively. Specifically, linkage analyses were performed on the ammonium oxalate, sodium carbonate, and $1 \mathrm{M} \mathrm{KOH}$ extracts from PdGAUT12.1-KD lines AB30.1 and AB30.8 and compared with WT Populus (Table 5). The PdGAUT12.1KD plants had 42 to $82 \%$ reduced levels of $4-\mathrm{GalA} p$ and 55 to $64 \%$ reduced amounts of terminal-GalA $p$ in the oxalate fractions compared with WT. These same PdGAUT12.1-KD fractions, compared to WT, had a 29 to $43 \%$ reduction in 2-linked Rhap, a linkage found in RG-I. Since the mole percent of 4-linked GalA to 2linked Rha was approximately 7 (Table 5), taken together, these linkage data indicate that the PdGAUT12.1-KD oxalate fraction extracts had reduced amounts of HG and lesser reductions in RG-I. There was also an increase in 4-Xyl $p$, T-Man $p$, 4-Man $p$, 4Gal $p$, T-Glcp, and 4-Glcp contents in PdGAUT12.1-KD plants compared with WT.

In the carbonate fractions, we observed 21 to $44 \%$ reduced amounts of 4-GalA $p$ and 30 to $50 \%$ reduced levels of terminal-GalA $p$ in the PdGAUT12.1-KD lines compared to WT (Table 5). The 2-linked Rha $p$ content was also reduced by 32 to $48 \%$ in the carbonate extracts of PdGAUT12.1-KD lines compared to WT, although again, the 2-linked Rha was only 8 mole\% of the GalA content. There was a corresponding increase in the amount of 4-Xyl $p$ and 4-Glcp in the PdGAUT12.1-KD carbonate fraction. These data are consistent with the hypothesis that the Populus GAUT12.1 gene encodes a glycosyltransferase that makes a subfraction of HG.

A significant reduction of 34 to $46 \%$ in 4-Xyl $p$ content in the $1 \mathrm{M} \mathrm{KOH}$ extract of the PdGAUT12.1-KD line corroborates the results from the glycosyl residue composition analysis (Table 5) and indicates that the $1 \mathrm{M}$ $\mathrm{KOH}$ fraction of the PdGAUT12.1-KD plants had reduced amounts of xylan (4-Xylp). A 73 to $93 \%$ reduction in 4-GalAp content in the KD lines indicates a reduction in the amount of HG compared to WT. Also, a 50 to $67 \%$ reduction of 2 -GalAp and a 44 to $50 \%$ reduction in 3-Rha $p$ suggests a simultaneous reduction in the xylan reducing end sequence [39]. The reduced content of 4-Xylp in this $1 \mathrm{M} \mathrm{KOH}$ extract along with reduced amounts of 2-GalAp and 3-Rhap would be consistent with a role for GAUT12 in the addition of GalA into xylan reducing end oligosaccharide sequence in Populus. The greater mole percent reduction in 4-GalAp compared to 2-GalAp would be consistent with a role for GAUT12 in synthesizing a GalA-containing moiety, possibly HG, required for the formation of xylan containing the reducing end oligosaccharide or linking the xylan to another structure in the wall. These overall results show that GAUT12 knockdown leads to defects in pectin and xylan production in Populus.

\section{Glycome profiling of fractionated cell walls from PdGAUT12.1-KD plants}

In an effort to better understand the specific defects in xylan and pectin, glycome profiling of sequential extracts from WT and PdGAUT12.1-KD walls was performed. Glycome profiling is an ELISA-based technique in which monoclonal antibodies directed against distinct cell wall carbohydrate epitopes are used to identify differences in 
Table 4 Glycosyl residue composition of cell wall (AIR) fractions from stem of controls and PdGAUT12.1-KD plants

\begin{tabular}{|c|c|c|c|c|c|c|c|c|c|}
\hline & \multirow[b]{2}{*}{ Ara } & \multicolumn{8}{|c|}{ Sugar composition of cell wall fractions ( $\mathrm{mol} \% ; \pm$ SE standard error) } \\
\hline & & Rha & Fuc & Xyl & GlcA & GalA & Man & Gal & Glc \\
\hline \multicolumn{10}{|c|}{ Ammonium oxalate } \\
\hline WT & $20.9 \pm 1.0$ & $4.1 \pm 0.2$ & $0.7 \pm 0.02$ & $8.9 \pm 0.2$ & $0.6 \pm 0.01$ & $23.1 \pm 1.0$ & $11.4 \pm 0.9$ & $3.7 \pm 0.3$ & $26.8 \pm 1.2$ \\
\hline V Control-1 & $21.0 \pm 0.6$ & $4.9 \pm 0.3$ & $0.8 \pm 0.02$ & $8.3 \pm 0.4$ & $0.5 \pm 0.02$ & $23.2 \pm 1.1$ & $11.6 \pm 1.0$ & $3.6 \pm 0.2$ & $26.9 \pm 1.0$ \\
\hline AB30.1 & $18.5 \pm 0.4$ & $1.0 \pm 0.3^{* *}$ & $0.2 \pm 0.01$ & $10.2 \pm 0.5$ & $0.7 \pm 0.02$ & $14.2 \pm 1.3^{*}$ & $19.5 \pm 1.1^{*}$ & $5.6 \pm 0.3^{*}$ & $30.6 \pm 0.9^{*}$ \\
\hline AB30.3 & $17.7 \pm 0.6^{*}$ & $2.1 \pm 0.2^{*}$ & $0.4 \pm 0.01$ & $10.5 \pm 0.2^{*}$ & $0.7 \pm 0.01$ & $12.9 \pm 0.9^{* *}$ & $18.2 \pm 1.3^{*}$ & $6.0 \pm 0.1^{*}$ & $31.4 \pm 1.0^{*}$ \\
\hline AB30.8 & $19.8 \pm 0.8$ & $2.1 \pm 0.1^{*}$ & $0.4 \pm 0.01$ & $11.1 \pm 0.3^{*}$ & $0.8 \pm 0.01$ & $5.0 \pm 0.5^{* *}$ & $21.9 \pm 1.2^{*}$ & $6.2 \pm 0.3^{*}$ & $32.8 \pm 1.1^{*}$ \\
\hline AB30.11 & $19.3 \pm 0.6$ & $2.3 \pm 0.2^{*}$ & $0.4 \pm 0.02$ & $11.4 \pm 0.3^{*}$ & $0.8 \pm 0.01$ & $8.9 \pm 0.8^{* *}$ & $20.2 \pm 1.3^{*}$ & $7.1 \pm 0.2^{*}$ & $29.4 \pm 1.2$ \\
\hline \multicolumn{10}{|c|}{ Sodium carbonate } \\
\hline WT & $10.7 \pm 0.6$ & $6.0 \pm 0.2$ & $0.3 \pm 0.02$ & $23.0 \pm 0.2$ & $1.6 \pm 0.01$ & $19.3 \pm 0.5$ & $14.0 \pm 0.9$ & $7.0 \pm 0.3$ & $18.0 \pm 0.9$ \\
\hline V Control-1 & $11.1 \pm 0.4$ & $5.9 \pm 0.1$ & $0.5 \pm 0.02$ & $22.5 \pm 0.6$ & $1.5 \pm 0.02$ & $19.5 \pm 0.8$ & $15.0 \pm 0.8$ & $6.7 \pm 0.2$ & $17.9 \pm 0.7$ \\
\hline AB30.1 & $4.8 \pm 0.4^{*}$ & $2.7 \pm 0.2^{*}$ & $0.4 \pm 0.01$ & $24.2 \pm 0.5$ & $1.7 \pm 0.02$ & $16.0 \pm 0.3^{*}$ & $18.0 \pm 1.0$ & $5.8 \pm 0.3$ & $26.9 \pm 0.9^{*}$ \\
\hline AB30.3 & $7.3 \pm 0.3$ & $2.7 \pm 0.1^{*}$ & $0.3 \pm 0.01$ & $24.7 \pm 0.2$ & $1.7 \pm 0.01$ & $14.0 \pm 0.4^{*}$ & $17.2 \pm 0.6$ & $6.8 \pm 0.3$ & $25.0 \pm 1.0^{*}$ \\
\hline AB30.8 & $7.9 \pm 0.3$ & $3.7 \pm 0.2^{*}$ & $0.8 \pm 0.01$ & $26.2 \pm 0.3$ & $2.6 \pm 0.01$ & $11.2 \pm 0.5^{*}$ & $19.3 \pm 0.9$ & $6.7 \pm 0.4$ & $21.9 \pm 1.1$ \\
\hline AB30.11 & $7.3 \pm 0.4$ & $3.4 \pm 0.3^{*}$ & $0.4 \pm 0.02$ & $25.7 \pm 0.3$ & $1.9 \pm 0.01$ & $13.5 \pm 0.4^{*}$ & $16.3 \pm 0.7$ & $7.4 \pm 0.2$ & $24.2 \pm 1.2^{*}$ \\
\hline \multicolumn{10}{|l|}{$1 \mathrm{M} \mathrm{KOH}$} \\
\hline WT & $0.5 \pm 0.04$ & $4.2 \pm 0.2$ & $0.2 \pm 0.02$ & $69.3 \pm 1.9$ & $8.5 \pm 0.3$ & $3.3 \pm 0.1$ & $6.4 \pm 0.6$ & $1.7 \pm 0.2$ & $7.4 \pm 1.2$ \\
\hline V Control-1 & $0.6 \pm 0.03$ & $4.1 \pm 0.1$ & $0.3 \pm 0.01$ & $70.1 \pm 2.3$ & $8.7 \pm 0.2$ & $3.4 \pm 0.2$ & $6.0 \pm 0.5$ & $1.6 \pm 0.3$ & $7.1 \pm 1.4$ \\
\hline AB30.1 & $1.1 \pm 0.05$ & $3.1 \pm 0.2$ & $0.4 \pm 0.01$ & $44.8 \pm 2.3^{*}$ & $5.5 \pm 0.2^{*}$ & $1.3 \pm 0.09^{*}$ & $10.7 \pm 0.5^{*}$ & $4.1 \pm 0.3^{* *}$ & $29.3 \pm 2.1^{* *}$ \\
\hline AB30.3 & $1.6 \pm 0.06$ & $3.1 \pm 0.1$ & $0.4 \pm 0.01$ & $42.4 \pm 2.4^{*}$ & $5.3 \pm 0.3^{*}$ & $1.2 \pm 0.1^{*}$ & $11.1 \pm 1.0^{*}$ & $3.7 \pm 0.1^{* *}$ & $31.9 \pm 2.5^{* *}$ \\
\hline AB30.8 & $1.8 \pm 0.1$ & $2.6 \pm 0.1^{*}$ & $0.5 \pm 0.01$ & $37.8 \pm 2.6^{* *}$ & $4.7 \pm 0.4^{*}$ & $0.8 \pm 0.08^{* *}$ & $14.6 \pm 0.8^{*}$ & $4.8 \pm 0.2^{* *}$ & $33.2 \pm 2.1^{* *}$ \\
\hline AB30.11 & $1.6 \pm 0.06$ & $3.3 \pm 0.2$ & $0.4 \pm 0.02$ & $38.4 \pm 3.2^{* *}$ & $4.8 \pm 0.3^{*}$ & $1.0 \pm 0.1^{* *}$ & $11.2 \pm 0.4^{*}$ & $3.8 \pm 0.2^{* *}$ & $35.9 \pm 2.4^{* *}$ \\
\hline \multicolumn{10}{|l|}{$4 \mathrm{M} \mathrm{KOH}$} \\
\hline WT & $2.3 \pm 0.08$ & $3.0 \pm 0.1$ & $0.5 \pm 0.02$ & $37.8 \pm 1.3$ & $4.7 \pm 0.2$ & $0.8 \pm 0.04$ & $11.9 \pm 1.2$ & $7.8 \pm 0.3$ & $31.2 \pm 1.2$ \\
\hline V Control-1 & $2.4 \pm 0.06$ & $3.2 \pm 0.2$ & $0.3 \pm 0.01$ & $38.1 \pm 1.5$ & $4.9 \pm 0.2$ & $0.9 \pm 0.03$ & $11.4 \pm 0.5$ & $7.9 \pm 0.3$ & $30.8 \pm 1.5$ \\
\hline AB30.1 & $1.6 \pm 0.09$ & $2.4 \pm 0.1$ & $0.4 \pm 0.01$ & $26.2 \pm 1.7^{*}$ & $3.4 \pm 0.2^{*}$ & $0.5 \pm 0.03^{*}$ & $18.2 \pm 0.5^{* *}$ & $8.4 \pm 0.3$ & $38.9 \pm 1.8^{*}$ \\
\hline AB30.3 & $2.1 \pm 0.07$ & $2.2 \pm 0.2$ & $0.3 \pm 0.01$ & $25.2 \pm 1.1^{*}$ & $3.3 \pm 0.3^{*}$ & $0.4 \pm 0.02^{*}$ & $15.6 \pm 1.0^{*}$ & $10.9 \pm 0.5^{* *}$ & $39.9 \pm 1.5^{*}$ \\
\hline AB30.8 & $1.7 \pm 0.05$ & $1.9 \pm 0.2^{*}$ & $0.3 \pm 0.01$ & $22.3 \pm 1.3^{* *}$ & $2.7 \pm 0.4^{*}$ & $0.3 \pm 0.03^{* *}$ & $18.9 \pm 0.8^{* *}$ & $8.6 \pm 0.6^{*}$ & $43.2 \pm 1.3^{* *}$ \\
\hline AB30.11 & $1.9 \pm 0.09$ & $2.3 \pm 0.1$ & $0.4 \pm 0.02$ & $23.2 \pm 1.4^{* *}$ & $2.9 \pm 0.3^{*}$ & $0.3 \pm 0.05^{* *}$ & $15.9 \pm 0.4^{*}$ & $8.1 \pm 0.2$ & $44.9 \pm 1.9^{* *}$ \\
\hline \multicolumn{10}{|l|}{ Chlorite } \\
\hline WT & $7.2 \pm 0.5$ & $4.3 \pm 0.1$ & - & $10.9 \pm 0.5$ & 0 & $6.4 \pm 0.2$ & $2.1 \pm 0.1$ & $11.2 \pm 0.4$ & $57.9 \pm 1.1$ \\
\hline V Control-1 & $7.0 \pm 0.3$ & $4.4 \pm 0.1$ & $0.2 \pm 0.02$ & $11.1 \pm 0.7$ & 0 & $6.5 \pm 0.3$ & $2.2 \pm 0.09$ & $10.9 \pm 0.5$ & $57.6 \pm 1.0$ \\
\hline AB30.1 & $5.5 \pm 0.3^{*}$ & $2.7 \pm 0.2^{*}$ & - & $7.2 \pm 0.4^{*}$ & 0 & $4.9 \pm 0.1^{*}$ & $3.0 \pm 0.1^{* *}$ & $13.6 \pm 0.6^{*}$ & $63.1 \pm 0.9^{*}$ \\
\hline
\end{tabular}


Table 4 Glycosyl residue composition of cell wall (AIR) fractions from stem of controls and PdGAUT12.1-KD plants (Continued)

\begin{tabular}{|c|c|c|c|c|c|c|c|c|c|}
\hline AB30.3 & $5.1 \pm 0.3^{*}$ & $2.7 \pm 0.3^{*}$ & - & $7.0 \pm 0.5^{*}$ & 0 & $4.6 \pm 0.2^{*}$ & $3.2 \pm 0.08^{* *}$ & $14.5 \pm 0.3^{*}$ & $63.6 \pm 1.2^{*}$ \\
\hline AB30.8 & $4.5 \pm 0.4^{*}$ & $3.7 \pm 0.2^{*}$ & - & $5.8 \pm 0.6^{* *}$ & 0 & $3.4 \pm 0.3^{*}$ & $3.1 \pm 0.09^{* *}$ & $14.2 \pm 0.4^{*}$ & $64.9 \pm 1.4^{*}$ \\
\hline AB30.11 & $4.4 \pm 0.3^{*}$ & $3.4 \pm 0.1^{*}$ & $0.4 \pm 0.01$ & $6.2 \pm 0.7^{*}$ & 0 & $4.3 \pm 0.2^{*}$ & $3.6 \pm 0.1^{* *}$ & $14.1 \pm 0.5^{*}$ & $63.7 \pm 0.9^{*}$ \\
\hline \multicolumn{10}{|l|}{$4 \mathrm{M} \mathrm{KOH} \mathrm{PC}$} \\
\hline WT & $1.1 \pm 0.05$ & $2.5 \pm 0.10$ & - & $65.1 \pm 2.1$ & $7.9 \pm 0.2$ & $2.4 \pm 0.06$ & $6.2 \pm 1.2$ & $5.0 \pm 0.8$ & $10.1 \pm 0.9$ \\
\hline V Control-1 & $1.3 \pm 0.06$ & $2.4 \pm 0.09$ & - & $64.6 \pm 2.3$ & $7.8 \pm 0.2$ & $2.5 \pm 0.07$ & $6.3 \pm 1.0$ & $5.2 \pm 0.6$ & $10.3 \pm 1.0$ \\
\hline AB30.1 & $1.1 \pm 0.04$ & $2.4 \pm 0.08$ & - & $47.5 \pm 1.9^{*}$ & $5.9 \pm 0.2^{*}$ & $2.2 \pm 0.06$ & $13.2 \pm 1.3^{*}$ & $9.1 \pm 0.7^{*}$ & $18.8 \pm 1.1^{*}$ \\
\hline AB30.3 & $1.0 \pm 0.03$ & $2.2 \pm 0.06$ & - & $42.3 \pm 1.5^{*}$ & $5.2 \pm 0.3^{*}$ & $2.1 \pm 0.06$ & $14.6 \pm 1.4^{* *}$ & $10.7 \pm 0.9^{* *}$ & $21.8 \pm 1.2^{*}$ \\
\hline AB30.8 & $0.9 \pm 0.05$ & $2.1 \pm 0.07$ & - & $35.7 \pm 1.8^{* *}$ & $4.3 \pm 0.2^{* *}$ & $2.0 \pm 0.08$ & $17.9 \pm 1.7^{* *}$ & $12.4 \pm 1.0^{* *}$ & $24.6 \pm 1.3^{*}$ \\
\hline AB30.11 & $1.0 \pm 0.05$ & $2.2 \pm 0.09$ & - & $37.8 \pm 1.6^{* *}$ & $4.7 \pm 0.3^{* *}$ & $2.0 \pm 0.09$ & $18.5 \pm 0.8^{* *}$ & $11.8 \pm 0.5^{* *}$ & $22.2 \pm 0.9^{*}$ \\
\hline
\end{tabular}

The amount of sugar is represented as average mol\% in each wall extract. AIR (alcohol insoluble residue) was sequentially extracted using $50 \mathrm{mM}$ ammonium oxalate, $50 \mathrm{mM} \mathrm{Na} \mathrm{CO}_{3}, 1 \mathrm{M} \mathrm{KOH}, 4 \mathrm{M} \mathrm{KOH}, 100 \mathrm{mM} \mathrm{sodium}$ chlorite (chlorite) and $4 \mathrm{M} \mathrm{KOH} \mathrm{PC} \mathrm{(post} \mathrm{chlorite),} \mathrm{hydrolyzed} \mathrm{with} \mathrm{methanolic} \mathrm{HCl}$, and analyzed by GC. Data are mean $\pm \mathrm{SE}$ of three biological and two technical replicates. Ital values represent significant difference between WT and $P d G A U T 12.1$-KD plants at ${ }^{*} P \leq 0.05$, ** $P \leq 0.001$ significance level (one-way ANOVA followed by Tukey's multiple comparison test). A dash designates that the amount of sugar was below detection limits. 
Table 5 Glycosyl linkage analysis of fractionated cell wall fractions from wild type $P$. deltoides and PdGAUT12.1-KD lines

\begin{tabular}{|c|c|c|c|c|c|c|c|c|c|}
\hline \multirow[t]{2}{*}{ Fractions } & \multicolumn{3}{|c|}{ Ammonium oxalate soluble } & \multicolumn{3}{|c|}{$\mathrm{Na}_{2} \mathrm{CO}_{3}$ soluble } & \multicolumn{3}{|c|}{$1 \mathrm{M} \mathrm{KOH}$ soluble } \\
\hline & WT & AB30.1 & AB30.8 & WT & AB30.1 & $\mathrm{AB} 30.8$ & WT & AB30.1 & AB30.8 \\
\hline t-Araf & 3.6 & 1.5 & 3.3 & 3.9 & 1.2 & 2.6 & 0.2 & 0.6 & 0.9 \\
\hline $\mathrm{t}$-Arap & 0.2 & 0.2 & 0.3 & 0.2 & 0.2 & 0.2 & 0.1 & 0.1 & 0.1 \\
\hline 3-Araf & 0.5 & 0.5 & 1.0 & 0.6 & 0.3 & 0.5 & - & 0.2 & 0.2 \\
\hline 3-Arap or 5-Araf & 3.2 & 2.4 & 2.5 & 2.2 & 0.8 & 1.3 & 0.2 & 0.2 & 0.6 \\
\hline 3,4-Arap or 3,5-Araf & 1.5 & 1.3 & 2.1 & - & - & - & - & - & - \\
\hline t-Rhap & 0.9 & 0.6 & 1.4 & 1.9 & 1.0 & 1.4 & 0.2 & 0.4 & 0.6 \\
\hline 2-Rhap & 2.1 & 1.2 & 1.5 & 3.1 & 1.6 & 2.1 & 0.7 & 0.5 & 0.4 \\
\hline 3-Rhap & - & - & - & - & - & - & 1.6 & 0.9 & 0.8 \\
\hline 2,4-Rhap & 1.1 & 1.0 & 1.4 & 0.8 & 0.7 & 0.8 & 0.5 & 1.0 & 1.0 \\
\hline t-Fucp & 0.3 & 0.2 & 0.4 & 0.6 & 0.4 & 0.8 & 0.2 & 0.5 & 0.5 \\
\hline $\mathrm{t}-\mathrm{Xylp}$ & 0.4 & 0.4 & 0.9 & 1.6 & 1.3 & 1.8 & 0.9 & 0.6 & 0.8 \\
\hline 4-Xylp & 6.5 & 9.0 & 12.5 & 20.5 & 21.8 & 22.1 & 59.3 & 38.9 & 32.3 \\
\hline 2,4-Xylp & 0.9 & 1.3 & 1.5 & 1.5 & 1.6 & 1.6 & 9.2 & 5.3 & 4.1 \\
\hline t-Manp & 1.2 & 1.2 & 2.2 & 1.8 & 1.8 & 2.0 & - & - & - \\
\hline 4-Manp & 17.8 & 22.9 & 19.2 & 19.6 & 20.1 & 20.9 & 6.0 & 11.0 & 15.0 \\
\hline 4,6-Manp & 0.7 & 0.9 & 0.5 & - & - & - & - & - & - \\
\hline $\mathrm{t}-\mathrm{GlcAp}$ & - & - & 2.0 & 1.6 & 1.7 & 2.3 & 8.5 & 6.8 & 3.7 \\
\hline 2-GlcAp & 0.1 & 0.2 & 0.2 & 0.2 & 0.2 & 0.2 & - & - & - \\
\hline $\mathrm{t}-\mathrm{Gal} A p$ & 1.1 & 0.4 & 0.5 & 2.0 & 1.0 & 1.4 & - & - & - \\
\hline 2-GalAp & - & - & 0.2 & 0.9 & 0.7 & 1.0 & 1.8 & 0.9 & 0.6 \\
\hline 4-GalAp & 23.8 & 13.7 & 4.2 & 15.5 & 12.3 & 8.7 & 1.5 & 0.4 & 0.1 \\
\hline 3,4-GalAp & - & - & - & 0.4 & - & 0.7 & - & - & - \\
\hline 2,4-GalAp & 0.1 & 0.1 & 0.1 & 0.1 & - & - & - & - & 0.1 \\
\hline $\mathrm{t}-\mathrm{Galp}$ & 2.9 & 2.1 & 2.7 & 3.2 & 3.1 & 3.0 & 1.2 & 2.9 & 3.1 \\
\hline 2-Galp & 0.4 & 0.2 & 0.3 & 0.6 & - & - & - & 0.4 & 0.6 \\
\hline 4-Galp & - & - & 3.3 & - & - & 2.8 & - & - & 0.1 \\
\hline 6-Galp & 0.4 & 1.1 & 0.8 & - & - & 0.8 & 0.5 & 0.8 & 1.0 \\
\hline 3,4-Galp & - & 1.2 & 1.0 & 0.3 & - & - & - & - & - \\
\hline 2,4-Galp & 0.2 & 0.3 & 0.3 & 0.2 & - & - & - & 0.1 & 0.2 \\
\hline $\mathrm{t}-\mathrm{Gl} p p$ & 3.3 & 3.4 & 4.3 & 3.6 & 2.9 & 3.2 & - & 2.6 & 2.8 \\
\hline $2-G \mid c p$ & 0.3 & 0.3 & 0.5 & 0.1 & 0.1 & 0.2 & - & - & - \\
\hline 4-Glcp & 26.2 & 31.6 & 28.3 & 12.9 & 25.5 & 17.7 & 7.4 & 24.4 & 29.8 \\
\hline $4,6-G \mid c p$ & 0.4 & 0.7 & 0.6 & - & - & - & - & 0.4 & 0.7 \\
\hline
\end{tabular}

All numbers are mole percentages. A dash designates that the amount of sugar was below detection limits. Glycosyl linkage analyses were provided by the CCRC Analytical Services.

specific carbohydrate structures present in sequential cell wall extracts [54-56]. Glycome profiling using a panel of 155 monoclonal antibodies directed against diverse cell wall matrix polysaccharide epitopes (Additional file 8) revealed changes in the extractability of three types of wall polysaccharides (Figure 9). In general, a marginal increase in the amounts of $1 \mathrm{M} \mathrm{KOH}$ extractable material (as noted in the bar graphs above the heat maps) was observed for all PdGAUT12.1-KD lines, suggesting an enhanced extractability of some hemicelluloses in these lines compared to the WT. A marginally enhanced abundance of xylan epitopes (recognized by the xylan- 6 and $7 \mathrm{mAb}$ groups) was observed in carbonate extracts from two GAUT12-KD lines (AB30.8 and AB30.11), suggesting that a portion of cell wall xylan is more easily extracted in the GAUT12-KD lines. Concomitantly, these two lines exhibited reduced abundance of these xylan epitopes in 


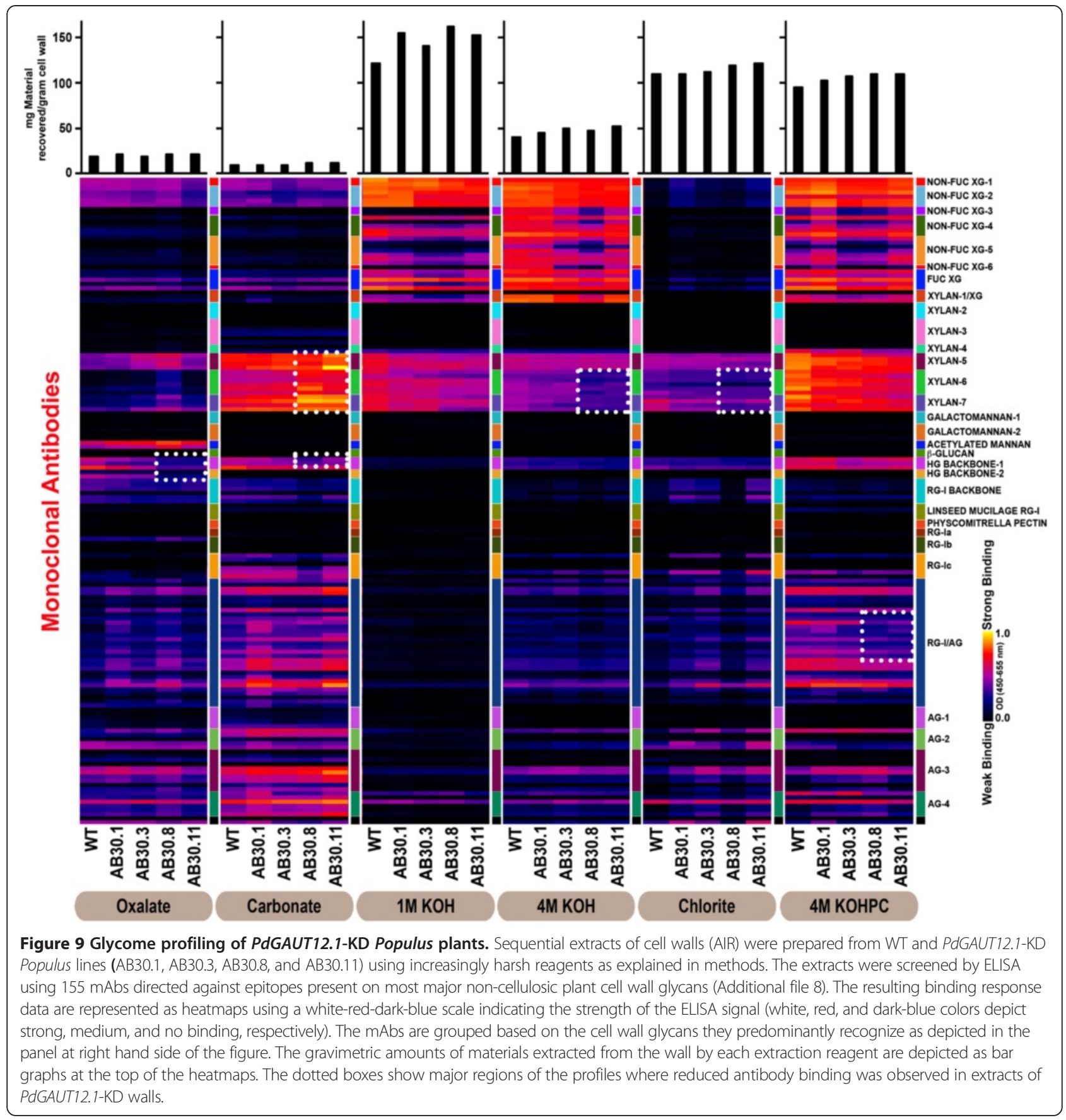

the $4 \mathrm{M} \mathrm{KOH}$ and chlorite extracts and a lesser reduction in the $1 \mathrm{M} \mathrm{KOH}$ extract. These results suggest that a portion of xylan in the PdGAUT12.1-KD lines (AB30.8 and AB30.11) was held less tightly in the walls and was recovered in the carbonate fraction. In these PdGAUT12.1-KD lines, there was also a reduction in HG epitopes in the oxalate extract, carbonate, and $4 \mathrm{M}$ $\mathrm{KOH}$ postchlorite fractions, as well as a slight reduction of some RG-I/AG mAb binding in the $4 \mathrm{M} \mathrm{KOH}$ postchlorite extract.
The combination of results obtained from glycosyl residue and linkage composition analyses of total AIR together with sugar composition analyses, glycome profiling, and total yield of sequential wall extracts indicate the following. (1) The only sugars consistently and statistically reduced in total AIR from PdGAUT12.1-KD lines were xylose and GalA, suggesting that GAUT12 may function in the addition of one or both of these into one or more wall polymers. (2) The only sugars consistently and statistically reduced in all the wall fractions, both 
the pectin- and xylan-enriched fractions, were GalA and rhamnose. Combined with linkage data, these results suggest that a pectin-like HG or HG/RG-I structure is either synthesized by GAUT12, or is part of the polymer synthesized by GAUT12. The significant reduction in xylose content in the four hemicellulose-enriched wall extracts suggests that xylan or a xylan-containing moiety may also be either the, or a part of, the polymer synthesized by GAUT12. (3) GAUT12.1-KD walls are more easily extracted as evidenced by the reduced yield of insoluble AIR pellet and the recovery of increased amounts of material in the sequential cell wall extracts. These results strongly support the hypothesis that the structure(s) synthesized by GAUT12 are part of the basic wall infrastructure. (4) Glycome profiling confirms that both xylan and HG along with some rhamnogalacturonan/arabinogalactan epitopes are affected in the PdGAUT12.1-KD lines, with losses in the HG and RG/ AG epitopes and an increased ease of extraction of the xylan epitope.

\section{Conclusions}

We applied a genetic engineering approach to modify GAUT12.1 expression in Populus. Eleven transgenic lines were obtained that had reduced PdGAUT12.1 transcript expression and an accompanying 25 to $47 \%$ reduction in GalA, 17 to $30 \%$ reduction in xylan content, and 3 to $7 \%$ increase in sugar release across the lines. We also found a significant increase in plant height (12 to $52 \%$ ) and stem diameter (12 to $44 \%$ ) and leaf size (52 to $117 \%)$.

It has been reported that removal of hemicellulose, especially xylose, xylo-oligomers, and xylan from lignocellulosic materials prior to enzymatic hydrolysis increases enzyme accessibility, conversion rates, and hydrolysis yield of plant biomass [41,42,57]. Similarly, pectin degradation has been shown to enhance saccharification [43]. Thus, a possible explanation for the greater sugar release in the P. deltoides PdGAUT12.1-KD lines is that the reduction of 17 to $30 \%$ xylose and 25 to $47 \%$ GalA associated with xylan and HG in the cell wall leads to an increased accessibility of the biomass to enzymes and results in greater conversion and sugar release. These data support our hypothesis that downregulation of GAUT12.1 reduces recalcitrance in Populus.

There was also a significant increase in phloem tissue, xylem fiber cell size, and xylem vessel diameter size in PdGAUT12.1-KD wood. The larger leaf area in both developing and expanded leaves, the larger individual palisade and spongy parenchyma cells, the higher RWC, and the increased phloem and wider vessels in the PdGAUT12.1-KD lines may explain the greater growth and biomass yield in PdGAUT12.1-KD RNAi Populus lines.
We also demonstrated that PdGAUT12.1, which is highly expressed in developing xylem (Figure 2B), can complement the phenotype of Arabidopsis irx 8 mutants (Figure 8), indicating that it is a functional homolog of the Arabidopsis GAUT12. PdGAUT12.2, which is expressed more broadly and at lower levels (Figure 2C), showed only partial complementation in the same experiments, suggesting that it might have functionally diverged from PdGAUT12.1.

The substantial decrease in GalA and xylose content in the PdGAUT12.1-KD cell walls and the content of fractionated walls as determined by glycosyl residue composition, linkage analyses, and glycome profiling support the hypothesis that reduced GAUT12.1 transcript levels affect both pectin and xylan biosynthesis in Populus. Sequential extraction of cell walls with increasingly harsh solvents in an effort to identify the specific polymers affected revealed an 11 to $17 \%$ increase in extractable material in walls of the PdGAUT12.1-KD lines compared to the controls and a concomitant 8 to $13 \%$ reduced mass of the final insoluble pellet, indicating a loosened wall structure in the PdGAUT12.1-KD lines that enables more facile extraction. Analyses of sequential cell wall extracts confirmed a reduction of both GX and the pectins HG and RG-I in extracts from PdGAUT12.1KD RNAi lines. These transgenic lines had a higher S/G ratio but no major change in the total lignin content. Since no major change in the total lignin was observed, the data support the hypothesis that GAUT12.1 synthesizes a wall structure that is either required for deposition of xylan and pectin or that contains xylan and pectin and that when this structure is not made, the wall is held together less tightly, leading to increased sugar release and increased growth. These results indicate that a systematic biotechnological manipulation of pectin and xylan biosynthetic genes in woody Populus can be used to develop less recalcitrant woody biomass with increased growth potential that can be more easily deconstructed and fermented into biofuels. The exact structure synthesized by GAUT12.1 remains to be determined, but the data presented here suggest it is a core architectural structure in Populus wood cell walls.

\section{Methods}

\section{Plant materials and growth conditions}

P. deltoides plants were grown in Fafards 3B Soil mix (GroSouth Inc, Atlanta, GA, USA) with osmocote $(250 \mathrm{ml} /$ bag $79 \mathrm{~L} \mathrm{3B}$ soil), bone meal $(84 \mathrm{ml} /$ bag $79 \mathrm{~L}$ 3B soil), gypsum ( $84 \mathrm{ml} /$ bag $79 \mathrm{~L} \mathrm{3B}$ soil), and dolomite/ limestone $(42 \mathrm{ml} / 1$ bag $3 \mathrm{~B}$ soil) in the greenhouse under a 16-h-light/8-h-dark cycle at 25 to $32^{\circ} \mathrm{C}$, depending on the season. All WT, vector control, and transgenic plants were grown for 9 months and fertilized weekly with Peters 20-10-20 (nitrogen-phosphorus-potassium; GroSouth Inc, 
Atlanta, GA, USA). The bottom $6 \mathrm{~cm}$ of the stem from WT, vector control, and transgenic 9-month-old plants was harvested and the bark removed by peeling with a razor. The peeled stem samples were air-dried and the pith was removed using a hand drill. The remaining stem material was milled to a particle size of 20 mesh $(0.85 \mathrm{~mm})$ using a Wiley Mini-Mill (model number 3383 L10, Thomas Scientific). Ground material was used for analytical pyrolysis, saccharification, and cell wall composition analyses.

Arabidopsis plants were grown on soil in a controlledenvironment chamber (Conviron, Pembina, ND, USA) under a 14 -h-light $/ 10$-h-dark cycle at $19^{\circ} \mathrm{C}$ during the light and $15^{\circ} \mathrm{C}$ during the dark. The light intensity was $150 \mu \mathrm{Em}^{-2} / \mathrm{s}$ and relative humidity was maintained at $50 \%$. T-DNA insertions were confirmed using primers from genomic regions flanking the T-DNA and the general T-DNA left border primer (Additional file 5). Arabidopsis Col-0 and heterozygous irx8-5 (SALK_044387) plants were transformed via the floral dip method [58], and transgenic plants harboring BASTA-resistant plasmids were selected on MS media plates containing $10 \mathrm{mg} / \mathrm{l}$ DL-phosphinothricin (PhytoTechnology Laboratories G523) and $100 \mathrm{mg} / \mathrm{l}$ timentin (PhytoTechnology Laboratories T869). Timentin inhibits residual Agrobacterium growth resulting from the dipping transformation. T1 transgenic plants harboring the construct in Col-O and irx8-5 homozygote mutant backgrounds were selected by genotyping PCR (Additional file 5) and used for complementation analyses.

\section{Vector construction and transformation of Populus}

The PtGAUT12.1 RNAi construct used for transformation of $P$. deltoides was generated by cloning a 168-bp fragment from the 3'-untranslated region of POPTR_0001s44250/ Potri.001G416800, which was amplified via PCR from a $P$. trichocarpa cDNA library using the forward primer $5^{\prime}$ CACCCCCGGGGAGAGTTCTTCACAAATCCA-3' and the reverse primer $5^{\prime}$ - TCTAGAAAATCCATAACATGCT TAATTTCTC-3'. The integrity of the fragment was verified by DNA sequencing (ACGT, Inc., Wheeling, IL, USA) after cloning into the Gateway entry vector, pENTR/DTOPO (Life Technologies, Grand Island, NY, USA). The fragment was transferred to a binary Gateway ${ }^{\circ}$ destination plasmid, pAGSM552 (GenBank accession KP259613) using LR Clonase II recombination (Life Technologies). The resulting binary transformation vector, pAGW641 (GenBank accession KP259612), was transformed into $A$. tumefaciens strain EHA105 via electroporation. The RNAi cassette comprised the Arabidopsis UBIQUITIN3 promoter, inverted repeats of the PtGAUT12.1 fragment flanking a spacer, and the nopaline synthase terminator. The spacer was a 1.3-kb fragment of the Petunia hybrida chsA intron. P. deltoides genotype WV94 was transformed using a modified Agrobacterium-based method [59,60]. Shoots regenerated from isolated calli were tested using PCR to verify the presence of the GAUT12.1 construct.

\section{Generation of constructs for complementation of Arabidopsis irx8 mutant}

Arabidopsis GAUT12 coding sequence (CDS) was amplified from Arabidopsis cDNA using primers whose sequences are provided in Additional file 5. Poplar GAUT12 cDNAs were generated by reverse transcription-PCR as follows: approximately $1 \mu \mathrm{g}$ of RNA harvested from WT $P$. deltoides stems was reverse transcribed into first-strand cDNA with oligo-dT primers. This library was diluted tenfold and used as a template for amplifying the predicted coding sequences of PdGAUT12.1(POPTR_0001s44250, Phytozome 8.0/ Potri.001G416800, Phytozome 10.0) and PdGAUT12.2 (POPTR_0011s13600, Phytozome 8.0/Potri.011G132600, Phytozome 10.0) by a nested PCR with specific primers designed on the basis of the P. trichocarpa genome sequence available at Phytozome (http://www.phytozome. net/, http://phytozome.jgi.doe.gov/). PCR products were gel-purified and ligated into PGEM $^{\circ}$-T Easy vector (Promega, Madison, WI, USA) and confirmed by sequencing. BamHI and ApaI restriction sites included in the second-round primers were used for lacing the coding sequences downstream of the $35 \mathrm{~S}$ promoter of a T-DNA derived from pCAMBIA3300. T-DNAs were moved into Agrobacterium tumefaciens strain GV3101 by electroporation and the cultures used for plant transformation as described above.

\section{RNA isolation and quantitative real-time PCR}

Plant tissues used to isolate RNA for transcript analysis were $P$. deltoides young leaves, mature leaves, stems of 6 to 8 internodes, petioles pooled from internodes 1 and 2 , cambium samples lightly scraped from frozen peeled bark, and xylem samples scraped from debarked frozen stem. All of the tissues were ground to a fine powder in liquid nitrogen. Total RNA was isolated from 100 to $150 \mathrm{mg}$ frozen powder from three individual tissue samples using hexadecyl-trimethylammonium bromide (CTAB; 2\% CTAB, 2\% polyvinylpyrolidone, $100 \mathrm{mM}$ Tris- $\mathrm{HCl}, 25 \mathrm{mM}$ EDTA, $2 \mathrm{M} \mathrm{NaCl}, 0.5 \mathrm{~g}$ L21 spermidine, and $2 \% \beta$-mercaptoethanol, $\mathrm{pH}$ 8.0) [61] and purified using columns from an RNeasy Plant Mini Kit (Qiagen, Venlo, Netherlands; 74903). Genomic DNA was removed by treatment with DNase (Ambion) and firststrand cDNA was synthesized from $1 \mu \mathrm{g}$ total RNA using SuperScript III First-Strand Synthesis Super Mix (Invitrogen). Primers for real-time PCR were designed using Beacon Designer (v 2.1, Premier Biosoft International, Palo Alto, CA, USA) and tested for efficiency and specificity. The least conserved region of the 
PtGAUT12.1 and PtGAUT12.2 genes in the poplar genome (Phytozome 8.0/Phytozome 10.0) was used for primer design. The primers used for this analysis were as follows: GAUT12.1 F 5'-GGTCGAGCAAAGCCTTGGCT AGATATAGC-3' and GAUT12.1 R 5' -AGATGGCCTAAT ATGACAGCCCTTTA-3' yielding a PCR product of 111 bp and GAUT12.2 F 5' - CATTTCAATGGTCGAGCA AAGCCTTGGC-3' and GAUT12.2 R 5' - GACAGCCCGT AATGAACTTGTCAGA-3' yielding a PCR product of $106 \mathrm{bp}$. The $18 S \mathrm{rRNA}$ reference gene was used to amplify a product of $74 \mathrm{bp}$ (18S F 5 '-AATTGTTGGTCTTCAACG AGGAA-3' and $18 S$ R $5^{\prime}$-AAAGGGCAGGGACGTAGTC AA-3'). The PCR reactions were performed in triplicate in a 96-well plate format using iQ TM SYBR Green Supermix (Bio-Rad, Hercules, CA, USA; 170-8882) in a CFX96 TM Real-Time PCR Detection System (Bio-Rad) according to the manufacturer's instructions. The relative transcript levels were calculated by normalizing target gene expression to control gene expression, where expression of the control gene was set to $1[62,63]$.

\section{Plant growth analysis, water status, and measurement of dry weight}

After the WT, vector control and transgenic plants developed proper roots in BTM media, all plants were transferred to soil in the greenhouse. Twelve to 15 plants per line were measured for plant height and radial stem growth. All plants were grown for nine months and initial plant stem growth was measured at three months. To determine the sizes of leaves from controls and transgenic lines at different developmental stages, the length and width of every fifth successive leaf from the apex was measured from three-month-old plants. Every $5^{\text {th }}, 6^{\text {th }}$ and $7^{\text {th }}$ leaf from the apex of six WT and six transgenic plants was collected and measured for leaf area to compare developing leaf growth. Similarly, the $20^{\text {th }}$ to $40^{\text {th }}$ leaves from the apex of five transgenic lines were collected and measured to compare fully expanded leaf areas.

Plant water status was measured as RWC of WT and PdGAUT12.1-KD plants [64]. The RWC is expressed as RWC $(\%)=[(\mathrm{FM}-\mathrm{DM}) /(\mathrm{TM}-\mathrm{DM})] \times 100$, where, FM, $\mathrm{DM}$, and TM are the fresh, dry, and turgid mass, respectively. Leaves were detached from the plants and used to measure FW. To obtain the turgid mass (TM), the detached leaves were rehydrated at $4^{\circ} \mathrm{C}$ for $24 \mathrm{~h}$. The rehydrated leaves were placed in a pre-heated oven at $70^{\circ} \mathrm{C}$ for $72 \mathrm{~h}$ to measure the dry mass $(\mathrm{DM})$. For the dry matter measurements, two sets of three individual three month old plants (above ground plant parts, that is, entire shoots) were harvested and each set was dried separately at $70^{\circ} \mathrm{C}$ for 5 days, weighed and mass values averaged $(N=6)$.

\section{Preparation of AIR cell wall and cell wall fractionations}

The ground biomass was sequentially extracted with $80 \%(v / v)$ ethanol, $100 \%$ ethanol, and chloroform/methanol $(1: 1[v / v])$, and after centrifugation, the resulting cell wall residue (AIR) was vacuum-dried for $24 \mathrm{~h}$ at room temperature. Sequential fractionations were carried out at $10 \mathrm{mg} / \mathrm{ml}$ based on the starting dry weight of AIR in order to isolate fractions enriched for different types of cell wall components. AIR wall was suspended at $25^{\circ} \mathrm{C}$ in $50 \mathrm{mM}$ ammonium oxalate, $\mathrm{pH} 5.0$ for $24 \mathrm{~h}$ with constant shaking at $100 \mathrm{rpm}$. After incubation, the mixture was centrifuged at $4,000 \mathrm{~g}$ for $15 \mathrm{~min}$ at room temperature and the supernatant saved as the ammonium oxalate extract. The residual pellet was subsequently washed three times with the same volume of deionized water, centrifuged, and the supernatant discarded. The pellet was treated at $25^{\circ} \mathrm{C}$ with $50 \mathrm{mM}$ $\mathrm{Na}_{2} \mathrm{CO}_{3}, \mathrm{pH} 10.0$ (containing $0.5 \%(w / v)$ sodium borohydride) with constant shaking (100 rpm). After centrifugation, the supernatant was saved as the sodium carbonate extract. The washed residual pellet was resuspended in $1 \mathrm{M} \mathrm{KOH}$ with $1 \%(w / v)$ sodium borohydride and incubated at $25^{\circ} \mathrm{C}$ for $24 \mathrm{~h}$ with constant shaking at $100 \mathrm{rpm}$. After incubation, the suspension was centrifuged at $4,000 \mathrm{~g}$ for $15 \mathrm{~min}$, the supernatant collected and labeled as $1 \mathrm{M} \mathrm{KOH}$ extract, and stored at $4^{\circ} \mathrm{C}$. The residual pellet from the $1 \mathrm{M} \mathrm{KOH}$ fraction was re-suspended in $4 \mathrm{M} \mathrm{KOH}$ with $1 \%(w / v)$ sodium borohydride and incubated at $25^{\circ} \mathrm{C}$ for $24 \mathrm{~h}$ with constant shaking at $100 \mathrm{rpm}$. After incubation, the suspension was centrifuged at $4,000 \mathrm{~g}$ for $15 \mathrm{~min}$ and the supernatant collected, labeled as $4 \mathrm{M} \mathrm{KOH}$ extract and stored at $4^{\circ} \mathrm{C}$. The residual pellet from the $4 \mathrm{M} \mathrm{KOH}$ fraction was treated with $100 \mathrm{mM}$ sodium chlorite at $70^{\circ} \mathrm{C}$ for $3 \mathrm{~h}$ to break down lignin polymers. After centrifugation, the supernatant was collected and labeled as chlorite extract. The residual pellet left after the sodium chlorite treatment was treated with $4 \mathrm{M} \mathrm{KOH}$ containing $1 \%(w /$ v) sodium borohydride extraction at $25^{\circ} \mathrm{C}$ for $24 \mathrm{~h}$ with constant shaking $(100 \mathrm{rpm})$ and the supernatant collected and labeled as $4 \mathrm{MKOH}$ PC. The $1 \mathrm{MKOH}$, $4 \mathrm{MKOH}$, and $4 \mathrm{MKOH} \mathrm{PC}$ fractions were neutralized using glacial acetic acid, dialyzed against six changes of de-ionized water at room temperature for $72 \mathrm{~h}$, and lyophilized [55].

\section{Glycosyl residue composition of AIR and fractionation of Populus stem cell walls}

Glycosyl residue composition analysis of stems from 9month-old WT, vector control, and PdGAUT12.1-KD plants was carried out by preparation and analysis of trimethylsilyl (TMS) derivatives of the monosaccharide methyl glycosides produced from the samples by acidic methanolysis [65,66]. The stem AIR (approximately 
$2 \mathrm{mg}$ ) and fractionated cell walls (100 to $300 \mu \mathrm{g}$ ) were aliquoted into individual tubes, and $20 \mu \mathrm{g}$ of inositol was added as an internal standard. The samples were lyophilized and dry samples hydrolyzed for $18 \mathrm{~h}$ at $80^{\circ} \mathrm{C}$ in $1 \mathrm{M}$ methanolic- $\mathrm{HCl}$. The samples were cooled, evaporated under a stream of dry air, and further dried two additional times with anhydrous methanol. The walls were derivatized with $200 \mu \mathrm{l}$ of TriSil Reagent (PierceEndogen, Rockford, IL, USA) and heated to $80^{\circ} \mathrm{C}$ for $20 \mathrm{~min}$. The cooled samples were evaporated under a stream of dry air, resuspended in $3 \mathrm{ml}$ hexane, and filtered through packed glass wool. The dried samples were resuspended in $150 \mu \mathrm{l}$ hexane and $1 \mu \mathrm{l}$ of sample was injected into the gas chromatograph-mass spectrometer (GC-MS). GC/MS analysis of the TMS methyl glycosides was performed on an Agilent 7890A GC interfaced to a 5975C MSD using a Supelco EC-1 fused silica capillary column $(30 \mathrm{~m} \times 0.25 \mathrm{~mm}$ ID) and helium as carrier gas.

\section{Glycosyl residue linkage analysis}

For glycosyl residue linkage analysis, the samples were permethylated, reduced, re-permethylated, depolymerized, reduced, and acetylated. The resulting partially methylated alditol acetates (PMAAs) were analyzed by gas chromatography-mass spectrometry (GC-MS) as previously described [66].

The fractionated wall samples from WT and PdGAUT12.1-KD lines were first suspended in $200 \mathrm{ul} \mathrm{di-}$ methyl sulfoxide and the samples stirred for 2 days at $25^{\circ} \mathrm{C}$. The samples were permethylated using potassium dimsyl anion and iodomethane. The permethylated uronic acids were reduced using lithium borodeuteride and then permethylated again by the method described earlier [67] with sodium hydroxide and methyl iodide in dry DMSO. This additional permethylation was to insure complete methylation of the polymer. Following sample workup, the permethylated material was hydrolyzed using $2 \mathrm{M}$ trifluoroacetic acid $\left(2 \mathrm{~h}\right.$ in sealed tube at $\left.121^{\circ} \mathrm{C}\right)$, reduced with $\mathrm{NaBD}_{4}$, and acetylated using acetic anhydride/TFA. The resulting PMAAs were analyzed on an Agilent 7890A GC interfaced to a 5975C MSD (mass selective detector, electron impact ionization mode). Separation was performed on a Supelco SP-2380 fused silica capillary column ( $30 \mathrm{~m} \times 0.25 \mathrm{~mm}$ ID).

\section{Analytical pyrolysis for lignin analysis}

All Populus biomass samples were analyzed by analytical pyrolysis for lignin content and S/G ratio. A commercially available molecular beam mass spectrometer (MBMS) designed specifically for biomass analysis was used for pyrolysis vapor analysis [68-70]. Approximately $4 \mathrm{mg}$ of air-dried 20-mesh biomass was introduced into the quartz pyrolysis reactor via $80 \mu \mathrm{l}$ deactivated stainless steel Eco-Cups provided with the autosampler. Mass spectral data from $\mathrm{m} / \mathrm{z} 30$ to 450 were acquired on a Merlin Automation data system version 3.0 using $17 \mathrm{eV}$ electron impact ionization. S/G ratios were determined by summing the syringyl peaks 154, 167, 168, $182,194,208$, and 210 and dividing by the sum of guaiacyl peaks 124, 137, 138, 150, 164, and 178 (Additional file 9). Several lignin peaks were omitted in the syringyl or guaiacyl summations due to individual peaks having associations with both $\mathrm{S}$ and $\mathrm{G}$ precursors [68]. Lignin estimates were determined by summing the intensities of the major lignin precursors listed in Additional file 9.

\section{Saccharification assay}

High-throughput thermochemical pretreatment and enzymatic hydrolysis of biomass was carried out as previously described [71,72]. Briefly, ground Populus biomass was extracted with glucoamylase (Spirizyme Ultra - $0.25 \%$ ) and alpha-amylase (Liquozyme SC DS $1.5 \%)$ in $0.1 \mathrm{M}$ sodium acetate $\left(24 \mathrm{~h}, 55^{\circ} \mathrm{C}, \mathrm{pH} 5.0\right)$ to remove possible starch content $(16 \mathrm{ml}$ enzyme solution per $1 \mathrm{~g}$ biomass) [73]. This was followed by an ethanol Soxhlet extraction for an additional $24 \mathrm{~h}$ to remove extractives. After drying overnight, $5 \mathrm{mg}( \pm 0.5 \mathrm{mg})$ was weighed in triplicate into individual wells of custommade 96-well format Hastelloy reactor plates. Water was added $(250 \mu \mathrm{l})$, the samples sealed with silicone adhesive-backed Teflon tape, clamped tightly, and treated at $180^{\circ} \mathrm{C}$ for $17.5 \mathrm{~min}$ in a customized steam reactor. Once cooled, $40 \mu \mathrm{l}$ of buffer-enzyme stock was added. The buffer-enzyme stock was $8 \%$ CTec2 (Novozymes) in $1.0 \mathrm{M}$ sodium citrate buffer. The samples were gently mixed and left to statically incubate at $50^{\circ} \mathrm{C}$ for $70 \mathrm{~h}$. After $70 \mathrm{~h}$ of incubation, an aliquot of the saccharified hydrolysate was diluted and analyzed using Megazyme's GOPOD (glucose oxidase/peroxidase) and XDH assays (xylose dehydrogenase), using mixed glucose/ xylose standards.

\section{Tissue fixation, embedding and microscopy}

Both leaf (6th leaf from the apex) and stem (10th and 20th internode from the apex) of $P$. deltoides tissues were cut into small pieces $(\sim 3 \mathrm{~mm})$ with a razor blade and immediately fixed in $25 \mathrm{mM}$ sodium phosphate buffer $\mathrm{pH}(7.1)$ with $4 \%(v / v)$ paraformaldehyde, $0.5 \%(v / v)$ glutaraldehyde, and $0.02 \%(v / v)$ Triton X-100 for $24 \mathrm{~h}$ at $4^{\circ} \mathrm{C}$. Tissues were washed three times for $30 \mathrm{~min}$ each with $25 \mathrm{mM}$ sodium phosphate buffer, $\mathrm{pH}$ 7.1, followed by three washes with water. In the next step, the samples underwent a graded ethanol series $(35 \%, 50 \%, 70 \%, 95 \%$, and $100 \%[v / v]$ ) wash for $25 \mathrm{~min}$ at each step (one time) to dehydrate the tissue. The $100 \%$ ethanol step was repeated two times to remove as much water as possible from the tissue. The samples were infiltrated with LR 
White embedding resin (Ted Pella) as follows: 1:3 (LR White: $100 \%$ ethanol) for $24 \mathrm{~h}$ (one time); 1:1 (LR White: $100 \%$ ethanol) for $24 \mathrm{~h}$ (one time); 3:1 (LR White: 100\% ethanol) for $24 \mathrm{~h}$ (one time); and LR White (100\%, no ethanol) for $24 \mathrm{~h}$ (three times). After the last resin change, the samples were transferred into gelatin capsules with fresh LR White. The gelatin capsules filled with resin were polymerized under 365-nm UV light for $48 \mathrm{~h}$ at $4^{\circ} \mathrm{C}$. Sections, approximately 1 um in thickness, were cut with a diamond Histo-knife on a Reichert-Jung Ultracut S ultramicrotome (Leica Microsystems, Wetzlar, Germany) and mounted onto pre-coated slides (Colorfrost/Plus, Fisher Scientific, Waltham, MA, USA). The mounted samples were stained with $1 \%$ toluidine blue for light microscopy. Images were captured using a Nikon DS-Ri1 camera (Nikon, Melville, NY, USA) and NISElements Basic Research software.

\section{Maceration of xylem}

The bottom part of the stem (approximately $6 \mathrm{~cm}$ ) from 9-month-old WT, vector control, and PdGAUT12.1-KD lines was harvested and debarked. Stem segments approximately $10 \mathrm{~mm}$ in length and $3 \mathrm{~mm}$ in diameter were used for maceration. The debarked stem segments were macerated with little modification in $50 \%$ glacial acetate acid and $3 \%$ hydrogen peroxide at $100^{\circ} \mathrm{C}$ for $12 \mathrm{~h}$ [74]. The samples were washed three times in water and the $\mathrm{pH}$ neutralized. The samples were suspended in $70 \%$ ethanol and vigorously shaken to release individual xylem fibers and vessel cells. The length and width of approximately 80 fibers and 60 vessel elements were measured from images captured using a Nikon DS-Ri1 camera (Nikon, Melville, NY) and NIS-Elements Basic Research software.

\section{Glycome profiling}

Glycome profiling of the sequential extracts prepared above was carried out as described previously [55]. Plant glycan-directed monoclonal antibodies [54-56] were from laboratory stocks (CCRC, JIM, and MAC series) at the Complex Carbohydrate Research Center (available through CarboSource Services; http://www. carbosource.net) or were obtained from BioSupplies (Australia) (BG1, LAMP). A description of the mAbs used in this study can be found in Additional file 8, which includes links to a web database, WallMAbDB (http://www.wallmabdb.net) that provides detailed information about each antibody.

\section{Statistical analysis}

Statistical analysis was performed using Statistica 5.0. The significance of differences between control and transgenic was analyzed using a one-way ANOVA followed by Tukey's multiple comparison test or post hoc Bonferroni corrected $t$-test.

\section{Additional files}

Additional file 1: Relationship of GAUT12.1 transcript expression to plant height and diameter.

Additional file 2: Physiological measurements on PdGAUT12.1-KD lines.

Additional file 3: Downregulation of GAUT12.1 leads to increased numbers of palisade parenchyma cells.

Additional file 4: Leaf anatomy of PdGAUT12.1-KD plants.

Additional file 5: List of primers used for complementation study. Additional file 6: Mass of extracts recovered upon extraction of PdGAUT12.1-KD AIR.

Additional file 7: Monosaccharide composition and total carbohydrate of cell wall fractions from PdGAUT12.1-KD and control plants.

Additional file 8: List of cell wall glycan-directed monoclonal antibodies (mAbs) used for glycome profiling analyses.

Additional file 9: Lignin peak and precursor assignments of analytical pyrolysis mass spectra.

\section{Abbreviations}

AIR: Alcohol-insoluble residue; GalA: Galacturonic acid;

GAUT1: Galacturonosyltransferase1; GAUT12: Galacturonosyltransferase12; GlcA: Glucuronic acid; GT8: Glycosyltransferase 8; GX: Glucuronoxylan; HG: Homogalacturonan; MBMS: Molecular beam mass spectrometer; MeGIcA: Methyl glucuronic acid; RG: Rhamnogalacturonan;

TMS: Trimethylsilyl; WT: Wild type.

\section{Competing interests}

The strategy to produce improved biomass as described in this paper has been included in a patent application. Cassandra Collins and Kim Winkeler are employees of ArborGen Inc., a global provider of conventional and next generation plantation tree seedling products for the forestry industry.

\section{Authors' contributions}

$A B$ is responsible for all aspects of the study, oversaw plant growth, line selection, tissue handling, and distribution, identified analyses to be done, carried out plant molecular, physiological, and cell wall analyses, and wrote the manuscript. ZH designed and performed the irx8 complementation experiments, interpreted the results, and contributed to qRT-PCR analyses and writing of the manuscript. SP performed the glycome profiling. XY identified Populus genes based on provided Arabidopsis homolog and designed the DNA construct. KW performed the molecular cloning and produced the RNAi plasmid. CC performed Populus transformation and propagated transgenic plants. SSM and DR aided in growth and analysis of the plants. EAR performed microtome sectioning of leaf and stem resin-embedded tissues. IG-A and K.H. contributed to the identification of genes for transgenesis and growth of plants. RWS conducted saccharification assays. GBT carried out sample preparation and operation of the high-throughput recalcitrance pipeline, including data acquisition and analysis for sugar release values. AZ and EG coordinated analysis of samples through the BioEnergy Science Center (BESC) high-throughput molecular beam mass spectrometry (MBMS) and saccharification pipelines. WL helped design, carry out, and interpret the irx8 complementation of the study. MFD developed and provided leadership for the BESC MBMS pipeline. SRD provided leadership and oversight in developing the standardized saccharification high-throughput pipeline and provided data analysis. MGH helped design and interpret the glycome profiling study. DM conceived of the study, coordinated research, contributed to interpretation of results and experimental design, and helped draft and finalize the manuscript. All authors read and approved the final manuscript. 


\section{Acknowledgements}

We thank Lee Gunter for validation of constructs containing amplified gene targets from P. trichocarpa leaf cDNA libraries, Rick Nelson for directing the BESC transformation pipeline and critical review of the manuscript, Will Rottmann for overseeing the Populus transformation pipeline, Sheilah Dixon Huckabee for administrative assistance, and the CCRC Analytical Services for glycosyl residue linkage analysis. The authors also thank Crissa Doeppke, Melissa Glenn, Kimberly Mazza, Logan Schuster, and Kevin Cowley for preparation of samples for the HTP biomass recalcitrance pipeline and Breeanna R. Urbanowicz for providing the poplar cDNA. The generation of the CCRC series of plant cell wall glycan-directed monoclonal antibodies used in this work was supported by the US National Science Foundation Plant Genome Program (DBI-0421683 and IOS-0923992). The research was funded by The BioEnergy Science Center (BESC) Grant DE-PS02-06ER64304 and partially by the Department of Energy Center Grant DE-FG02-93ER20097. The BioEnergy Science Center is a U.S. Department of Energy Bioenergy Research Center supported by the Office of Biological and Environmental Research in the DOE Office of Science.

\section{Author details}

${ }^{1}$ Department of Biochemistry and Molecular Biology, University of Georgia, B122 Life Sciences Bldg., Athens, GA 30602, USA. 'Department of Plant Biology, University of Georgia, 2502 Miller Plant Sciences, Athens, GA 30602, USA. ${ }^{3}$ Complex Carbohydrate Research Center, University of Georgia, 315 Riverbend Road, Athens, GA 30602, USA. ${ }^{4}$ DOE-BioEnergy Science Center (BESC), Oak Ridge, USA. ${ }^{5}$ Bioscience Division, Oak Ridge National Laboratory, Oak Ridge, TN 37831, USA. ' ArborGen Inc., 2011 Broadbank Ct, Ridgeville, SC 29472, USA. ${ }^{7}$ National Renewable Energy Laboratory, 15013 Denver West Parkway, Golden, CO 80401-3305, USA.

Received: 16 September 2014 Accepted: 3 February 2015 Published online: 12 March 2015

\section{References}

1. Himmel ME, Ding SY, Johnson DK, Adney WS, Nimlos MR, Brady JW, et al. Biomass recalcitrance: engineering plants and enzymes for biofuels production. Science. 2007;315:804-7.

2. Somerville C, Youngs H, Taylor C, Davis SC, Long SP. Feedstocks for lignocellulosic biofuels. Science. 2010;329:790-2.

3. Pauly M, Keegstra K. Plant cell wall polymers as precursors for biofuels. Curr Opin Plant Biol. 2010;13:305-12.

4. Mellerowicz EJ, Baucher M, Sundberg B, Bojeran W. Unraveling cell wall formation in the woody dicot stem. Plant Mol Biol. 2001;47:239-74.

5. Hao Z, Mohnen D. A review of xylan and lignin biosynthesis: foundation for studying Arabidopsis irregular xylem mutants with pleiotropic phenotypes. Crit Rev Biochem Mol Biol. 2014;49:212-41.

6. Brown DM, Zeef LA, Ellis J, Goodacre R, Turner SR. Identification of novel genes in Arabidopsis involved in secondary cell wall formation using expression profiling and reverse genetics. Plant Cell. 2005;17:2281-95.

7. Lee C, O'Neill MA, Tsumuraya Y, Darvill AG, Ye ZH. The irregular xylem9 mutant is deficient in xylan xylosyltransferase activity. Plant Cell Physiol. 2007:48:1624-34

8. Pena MJ, Zhong RQ, Zhou GK, Richardson EA, O'Neill MA, Darvill AG, et al. Arabidopsis irregular xylem 8 and irregular xylem9: Implications for the complexiy of glucuronoxylan biosynthesis. Plant Cell. 2007;19:549-63.

9. Wu AM, Hornblad E, Voxeur A, Gerber L, Rihouey C, Lerouge P, et al. Analysis of the Arabidopsis IRX9/IRX9- $L$ and IRX14/IRX14- $L$ pairs of glycosyltransferase genes reveals critical contributions to biosynthesis of the hemicellulose glucuronoxylan. Plant Physiol. 2010;153:542-54.

10. Brown DM, Goubet F, Vicky WWA, Goodacre R, Stephens E, Dupree P, et al. Comparison of five xylan synthesis mutants reveals new insight into the mechanisms of xylan synthesis. Plant J. 2007;52:1154-68.

11. Keppler BD, Showalter AM. IRX14 and IRX14-LIKE, two glycosyl transferases involved in glucuronoxylan biosynthesis and drought tolerance in Arabidopsis. Mol Plant. 2010;3:834-41.

12. Brown DM, Zhang ZN, Stephens E, Dupree P, Turner SR. Characterization of IRX10 and IRX10-like reveals an essential role in glucuronoxylan biosynthesis in Arabidopsis. Plant J. 2009;57:732-46.

13. Wu AM, Rihouey C, Seveno M, Hornblad E, Singh SK, Matsunaga T, et al. The Arabidopsis IRX10 and IRX10-LIKE glycosyltransferases are critical for glucuronoxylan biosynthesis during secondary cell wall formation. Plant J. 2009;57:718-31.

14. Brown D, Wightman R, Zhang Z, Gomez LD, Atanassov I, Bukowski JP, et al Arabidopsis genes IRREGULAR XYLEM (IRX15) and IRX15L encode DUF579containing proteins that are essential for normal xylan deposition in the secondary cell wall. Plant J. 2011;66:401-13.

15. Jensen JK, Kim H, Cocuron JC, Orler R, Ralph J, Wilkerson CG. The DUF579 domain containing proteins IRX15 and IRX15-L affect xylan synthesis in Arabidopsis. Plant J. 2011;66:387-400.

16. Urbanowicz BR, Peña MJ, Moniz HA, Moremen KW, York WS. Two Arabidopsis proteins synthesize acetylated xylan in vitro. Plant J. 2014;80:197-206.

17. Jensen JK, Johnson NR, Wilkerson CG. Arabidopsis thaliana IRX10 and two related proteins from psyllium and Physcomitrella patens are xylan xylosyltransferases. Plant J. 2014;80:207-15.

18. Rennie EA, Hansen SF, Baidoo EE, Hadi MZ, Keasling JD, Scheller HV. Three members of the Arabidopsis glycosyltransferase family 8 are xylan glucuronosyltransferases. Plant Physiol. 2012;159:1408-17.

19. Mortimer JC, Miles GP, Brown DM, Zhang ZN, Segura MP, Weimar T, et al. Absence of branches from xylan in Arabidopsis gux mutants reveals potential for simplification of lignocellulosic biomass. Proc Natl Acad Sci U S A. 2010;107:17409-14.

20. Urbanowicz BR, Pena MJ, Ratnaparkhe S, Avci U, Backe J, Steet HF, et al. 4-O-Methylation of glucuronic acid in Arabidopsis glucuronoxylan is catalyzed by a Domain of Unknown Function family 579 protein. Proc Natl Acad Sci U S A. 2012;109:14253-8.

21. Zhong RQ, Pena MJ, Zhou GK, Nairn CJ, Wood-Jones A, Richardson EA, et al Arabidopsis fragile fiber8, which encodes a putative glucuronyltransferase, is essential for normal secondary wall synthesis. Plant Cell. 2005;17:3390-408.

22. Lee C, Teng Q, Huang WL, Zhong RQ, Ye ZH. The Poplar GT8E and GT8F glycosyltransferases are functional orthologs of Arabidopsis PARVUS involved in glucuronoxylan biosynthesis. Plant Cell Physiol. 2009;50:1982-7.

23. Bouton S, Leboeuf E, Mouille G, Leydecker MT, Talbotec J, Granier F, et al. QUASIMODO1 encodes a putative membrane-bound glycosyltransferase required for normal pectin synthesis and cell adhesion in Arabidopsis. Plant Cell. 2002;14:2577-90.

24. Orfila C, Sørensen SO, Harholt J, Geshi N, Crombie H, Truong HN, et al. QUASIMODO1 is expressed in vascular tissue of Arabidopsis thaliana inflorescence stems, and affects homogalacturonan and xylan biosynthesis. Planta. 2005;222:613-22.

25. Leboeuf E, Guillon F, Thoiron S, Lahaye M. Biochemical and immunohistochemical analysis of pectic polysaccharides in the cell walls of Arabidopsis mutant QUASIMODO 1 suspension-cultured cells: implications for cell adhesion. J Exp Bot. 2005:56:3171-82.

26. Shao M, Zheng H, Hu Y, Liu D, Jang JC, Ma H, et al. The GAOLAOZHUANGREN1 gene encodes a putative glycosyltransferase that is critical for normal development and carbohydrate metabolism. Plant Cell Physiol. 2004;45:1453-60.

27. Lee C, Zhong R, Richardson E, Himmelsbach D, McPhail B, Ye ZH. The PARVUS gene is expressed in cells undergoing secondary wall thickening and is essential for glucuronoxylan biosynthesis. Plant Cell Physiol. 2007;48:1659-72

28. Kong Y, Zhou G, Avci U, Gu X, Jones C, Yin Y, et al. Two poplar glycosyltransferase genes, PdGATL1.1 and PdGATL1.2, are functional orthologs to PARVUS/AtGATL1 in Arabidopsis. Mol Plant. 2009;2:1040-50.

29. Persson S, Caffall KH, Freshour G, Hilley MT, Bauer S, Poindexter $P$, et al. The Arabidopsis irregular xylem 8 mutant is deficient in glucuronoxylan and homogalacturonan, which are essential for secondary cell wall integrity. Plant Cell. 2007;19:237-55.

30. Hao Z, Avci U, Tan L, Zhu X, Glushka J, Pattathil S, et al. Loss of Arabidopsis GAUT12/IRX8 causes anther indehiscence and leads to reduced $G$ lignin associated with altered matrix polysaccharide deposition. Frontiers Plant Sci. 2014;5:357.

31. Campbell JA, Davies GJ, Bulone V, Henrissat B. A classification of nucleotidediphospho-sugar glycosyltransferases based on amino acid sequence similarities. Biochem J. 1997;326:929-39.

32. Coutinho PM, Deleury E, Davies GJ, Henrissat B. An evolving hierarchical family classification for glycosyltransferases. J Mol Biol. 2003;328:307-17.

33. Yin $Y$, Chen $H$, Hahn MG, Mohnen D, Xu Y. Evolution and function of the plant cell wall synthesis-related Glycosyltransferase Family 8. Plant Physiol. 2010;153:1729-46. 
34. Sterling JD, Atmodjo MA, Inwood SE, Kumar Kolli VS, Quigley HF, Hahn MG, et al. Functional identification of an Arabidopsis pectin biosynthetic homogalacturonan galacturonosyltransferase. Proc Natl Acad Sci U S A. 2006;103:5236-41.

35. Atmodjo MA, Hao Z, Mohnen D. Evolving views of pectin biosynthesis. Annu Rev Plant Biol. 2013;64:747-79.

36. Atmodjo MA, Sakuragi Y, Zhu X, Burrell JA, Mohanty SS, Atwood III JA, et al. GAUT1:GAUT7 are the core of a plant cell wall pectin biosynthetic homogalacturonan:galacturonosyltransferase complex. Proc Natl Acad Sci U S A. 2011;108:20225-30.

37. Li Q, Min D, Wang JP-Y, Peszlen I, Horvath L, Horvath B, et al. Down-regulation of glycosyltransferase $8 \mathrm{D}$ genes in Populus trichocarpa caused reduced mechanical strength and xylan content in wood. Tree Physiol. 2011;31:226-36.

38. Zhou GK, Zhong R, Himmelsbach DS, McPhail BT, Ye ZH. Molecular characterization of PoGT8D and PoGT43B, two secondary wall-associated glycosyltransferases in poplar. Plant Cell Physiol. 2007:48:689-99.

39. Lee C, Teng Q, Zhong R, Ye ZH. Molecular dissection of xylan biosynthesis during wood formation in poplar. Mol Plant. 2011;4:730-47.

40. Olson DG, McBride JE, Shaw AJ, Lynd LR. Recent progress in consolidated bioprocessing. Curr Opin Biotechnol. 2012;23:396-405.

41. Shin HD, McClendon S, Vo T, Chen RR. Escherichia coli binary culture engineered for direct fermentation of hemicellulose to a biofuel. Appl Environ Microbiol. 2010;76:8150-9.

42. Qing Q, Yang B, Wyman CE. Xylooligomers are strong inhibitors of cellulose hydrolysis by enzymes. Bioresour Technol. 2010;101:9624-30.

43. Lionetti V, Francocci F, Ferrari S, Volpi C, Bellincampi D, Galletti R, et al. Engineering the cell wall by reducing de-methyl-esterified homogalacturonan improves saccharification of plant tissues for bioconversion. Proc Natl Acad Sci U S A. 2010;107:616-21.

44. Biswal AK, Soeno K, Gandla ML, Immerzeel P, Pattathil S, Lucenius J, et al. Aspen pectate lyase PtxtPL1-27 mobilizes matrix polysaccharides from woody tissues and improves saccharification yield. Biotechnol Biofuels. 2014;7:11.

45. Mohnen DA, Biswal AK, Hao Z, Kataeva I, Adams MW, Hunt KD, et al. Plants with altered cell wall biosynthesis and methods of use. PCT Application \# PCT/US20011/032733, filed on 4/15/2011, published on 10/20/2011 as WO 2011130666, and claiming priority to 4/16/2010.

46. Caffall KH, Pattathil S, Phillips SE, Hahn MG, Mohnen D. Arabidopsis thaliana T-DNA mutants implicate GAUT genes in the biosynthesis of pectin and xylan in cell walls and seed testa. Mol Plant. 2009;2:1000-14.

47. Tamura K, Peterson D, Peterson N, Stecher G, Nei M, Kumar S. MEGA5: molecular evolutionary genetics analysis using maximum likelihood, evolutionary distance, and maximum parsimony methods. Mol Biol Evol. 2011:28:2731-9.

48. Chalupa V. Clonal propagation of broad-leaved forest trees in vitro. Commun Inst Forest Cechosl. 1981;12:255-71.

49. Petersen PD, Lau J, Ebert B, Yang F, Verhertbruggen Y, Kim JS, et al. Engineering of plants with improved properties as biofuels feedstocks by vessel-specific complementation of xylan biosynthesis mutants. Biotechnol Biofuels. 2012;5:84

50. Studer MH, DeMartini JD, Davis MF, Sykes RW, Davison B, Keller M, et al. Lignin content in natural Populus variants affects sugar release. Proc Natl Acad Sci U S A. 2011;108:6300-5.

51. Van Acker R, Leplé JC, Aerts D, Storme V, Goeminne G, Ivens B, et al. Improved saccharification and ethanol yield from field-grown transgenic poplar deficient in cinnamoyl-CoA reductase. Proc Natl Acad Sci U S A. 2014;111:845-50.

52. Voelker SL, Lachenbruch B, Meinzer FC, Jourdes M, Ki C, Patten AM, et al. Antisense down-regulation of $4 \mathrm{CL}$ expression alters lignification, tree growth, and saccharification potential of field-grown poplar. Plant Physiol. 2010;154:874-86.

53. Kramer PJ, Boyer JS. Water relations of plants and soils. San Diego: Academic Press; 1995. p. 495.

54. Pattathil S, Avci U, Baldwin D, Swennes AG, McGill JA, Popper Z, et al. A comprehensive toolkit of plant cell wall glycan-directed monoclonal antibodies. Plant Physiol. 2010;153:514-25.

55. Pattathil S, Avci U, Miller JS, Hahn MG. Immunological approaches to plant cell wall and biomass characterization: glycome profiling. In: Himmel ME, editor. Biomass conversion. Methods and protocols (Springer), methods in molecular biology, vol, vol. 908. New York: Humana Press; 2012. p. 61-72.
56. DeMartini JD, Pattathil S, Avci U, Szekalski K, Mazumder K, Hahn MG, et al. Application of monoclonal antibodies to investigate plant cell wall deconstruction for biofuels production. Energy Environ Sci. 2011:4:4332-9.

57. Liao W, Wen ZY, Hurley S, Liu Y, Liu CB, Chen SL. Effects of hemicellulose and lignin on enzymatic hydrolysis of cellulose from dairy manure. Appl Biochem Biotechnol. 2005;2005(121):1017-30.

58. Clough SJ, Bent AF. Floral dip: a simplified method for Agrobacterium-mediated transformation of Arabidopsis thaliana. Plant J. 1998;16:735-43.

59. Tsai CJ, Podila GK, Chiang VL. Agrobacterium-mediated transformation of quaking aspen (Populus tremuloides) and regeneration of transgenic plants. Plant Cell Rep. 1994;14:94-7.

60. Mingozzi M, Montello P, Merkle S. Adventitious shoot regeneration from leaf explants of eastern cottonwood (Populus deltoides) cultured under photoautotrophic conditions. Tree Physiol. 2009;29:333-43.

61. Chang SJ, Puryear J, Cairney J. A simple and efficient method for isolating RNA from pine trees. Plant Mol Biol Rep. 1993;11:113-6.

62. Livak KJ, Schmittgen TD. Analysis of relative gene expression data using real-time quantitative PCR and the $22^{-\triangle \Delta C T}$ method. Methods. 2001;25:402-8.

63. Brunner AM, Yakovlev IA, Strauss SH. Validating internal controls for quantitative plant gene expression studies. BMC Plant Biol. 2004;4:14.

64. Boyer JS. Measurement of the water status of plants. Annu Rev Plant Physiol. 1968;9:351-63.

65. Tan L, Eberhard S, Pattathil S, Warder C, Glushka J, Yuan C, et al. Arabidopsis cell wall proteoglycan consists of pectin and arabinoxylan covalently linked to an arabinogalactan protein. Plant Cell. 2013;25:270-87.

66. York W, Darvill AG, McNeil M, Stevenson TT, Albersheim P. Isolation and characterization of plant cell walls and cell wall components. Methods Enzymol. 1985;118:3-40.

67. Ciucanu I, Kerek F. A simple and rapid method for the permethylation of carbohydrates. Carbohydr Res. 1984;131:209-17.

68. Evans RJ, Milne TA. Molecular characterization of the pyrolysis of biomass. Energy Fuels. 1987;1:123-37.

69. Sykes R, Yung M, Novaes E, Kirst M, Peter G, Davis M. High-throughput screening of plant cell-wall composition using pyrolysis molecular beam mass spectroscopy. In: Mielenz JR, editor. Biofuels:methods and protocols, methods in molecular biology. New York: Humana Press; 2009. p. 169-83.

70. Tuskan G, West D, Bradshaw HD, Neale D, Sewell M, Wheeler N, et al. Two high-throughput techniques for determining wood properties as part of a molecular genetics analysis of hybrid poplar and loblolly pine. Appl Biochem Biotechnol. 1999;77:55-65.

71. Selig MJ, Tucker MP, Sykes RW, Reichel KL, Brunecky R, Himmel ME, et al. Biomass recalcitrance screening by integrated high throughput hydrothermal pretreatment and enzymatic saccharification. Ind Biotechnol. 2010;6:104-11.

72. Decker SR, Brunecky R, Tucker MP, Himmel ME, Selig MJ. High throughput screening techniques for biomass conversion. Bioenergy Res. 2009;2:79-192.

73. Decker SR, Carlile M, Selig MJ, Doeppke C, Davis M, Sykes R, et al. Reducing the effect of variable starch levels in biomass recalcitrance screening. In: Himmel ME, editor. Biomass conversion. Methods in molecular biology, vol, vol. 908. New York: Humana Press; 2012. p. 181-95.

74. Gray-Mitsumune M, Blomquist K, McQueen-Mason S, Teeri T, Sundberg B, Mellerowicz EJ. Ectopic expression of a wood-abundant expansin PttEXPA1promotes cell expansion in primary and secondary tissues in aspen. Plant Biotechnol J. 2008;6:62-72.

\section{Submit your next manuscript to BioMed Central and take full advantage of:}

- Convenient online submission

- Thorough peer review

- No space constraints or color figure charges

- Immediate publication on acceptance

- Inclusion in PubMed, CAS, Scopus and Google Scholar

- Research which is freely available for redistribution 